\title{
Machine Learning the Redox Potentials of
}

\section{Phenazine Derivatives: A Comparative Study on}

\section{Molecular Features}

\author{
Siddharth Ghule ${ }^{*,+,}$, Sayan Bagchi ${ }^{\dagger, *}$, Kumar Vanka*,+,* \\ $†$ Physical and Materials Chemistry Division, CSIR-National Chemical Laboratory (CSIR-NCL), \\ Dr. Homi Bhabha Road, Pashan, Pune 411008, India
}

†Academy of Scientific and Innovative Research (AcSIR), Ghaziabad 201002, India 


\section{ABSTRACT}

Redox Flow Batteries (RFBs) are promising candidates for green and efficient energy storage systems. However, Their widespread adoption still needs further investigations into cheaper and greener alternative organic redox-active species. In this work, we have developed machinelearning models to predict the redox potential of phenazine derivatives in DME (dimethoxyethane) solvent using a small dataset of 185 molecules. 2D, 3D, and molecular fingerprint features were computed using readily available and easy-to-use Python libraries, making our approach easily adaptable to similar work. Twenty linear and non-linear machinelearning models were investigated in this work. These models achieved excellent performance on the unseen data (i.e., $\mathrm{R}^{2}>0.98, \mathrm{MSE}<0.008 \mathrm{~V}^{2}$ and $\mathrm{MAE}<0.07 \mathrm{~V}$ ). Model performance was assessed consistently using the training and evaluation "pipeline" method developed in this work. We showed that 2D molecular features were most informative and achieved the best prediction accuracy among four feature sets. We also showed that often less preferred but relatively faster linear models could perform better than non-linear models when the feature set contains different types of features (i.e., 2D, 3D, and molecular fingerprints). Further investigations revealed that it is possible to reduce the training and inference time without sacrificing prediction accuracy by using a small subset of features. Moreover, models were able to predict the previously reported promising redox-active compounds with high accuracy. Also, significantly low prediction errors were observed for most functional groups. Thus, we believe the results obtained in this report would help in the adoption of green energy by accelerating the field of materials discovery for energy storage applications. 


\section{INTRODUCTION}

Today, $\sim 85 \%$ of the world's energy demand is being fulfilled by fossil fuels ${ }^{1,2}$. The limited supply of fossil fuels and the ever-increasing population has raised concerns that we might run out of fossil fuels sooner than expected. ${ }^{1,3}$ Furthermore, electricity production from fossil fuels is one of the major factors responsible for greenhouse gas emissions. ${ }^{4}$ In this age, humanity faces two major challenges: of balancing increased energy demand while reducing the environmental impact associated with energy production. In the past decades, investments and research efforts in green technology have been increased to overcome these challenges. ${ }^{5}$ Significant progress has already been made to access renewable energy sources. ${ }^{6,7}$ Renewable energy sources, being intermittent, require efficient energy storage. ${ }^{4}$ Improvements in the energy storage technology would not only help in the adoption of renewable energy but also help in making efficient use of non-renewable energy sources. Historically, it has been more expensive to store energy than to expand energy generation to handle increased demand. ${ }^{8}$ Thus, grid systems employed today are likely to fail when additional energy cannot be generated during peak demand. The massive Texas Blackout in February, 2021 is an example of such a failure. ${ }^{9}$ It suggests that efficient energy storage technology is urgently required. Unfortunately, only $1.0 \%$ of the energy consumed worldwide can be stored with the energy storage technology accessible today. ${ }^{10}$ Furthermore, the contribution of electrochemical batteries to energy storage capacity is less than $2.0 \%$, even though most of the devices we use every day include batteries ${ }^{8,10}$. Li-ion batteries are widely used today due to their high energy density, high specific energy, long cycle life, and fast charge-discharge cycle., ${ }^{4,8,11}$ Unfortunately, Li-ion batteries suffer from high production costs, safety issues, and high environmental impact. ${ }^{2,12}$ Redox Flow Batteries (RBFs) have the potential to overcome drawbacks of Li-ion batteries owing to their high storage capacity, 
independent control over storage capacity and power, fast responsiveness, ease of scaling, room temperature operation, cost-effectiveness, high round trip efficiency, safety, and negligible environmental impact. ${ }^{13-15}$ RBFs are increasingly being used as energy storage devices in renewable energy systems, thereby helping in the adoption of green energy. ${ }^{15,16}$ A schematic diagram of the typical redox flow battery is shown in Figure 1. RFB consists of two storage tanks containing cathode and anode redox-active species dissolved in an electrolyte solution. The electrolyte solution in the positive and negative compartments is termed catholyte and anolyte, respectively. These storage tanks are connected to an electrochemical cell (or current collector) via pumps. The electrochemical cell consists of porous electrodes separated by an ion-selective membrane. During operation, electrolytes containing redox-active species are pumped to the electrochemical cell, where redox-active species undergo either oxidation or reduction depending on the charge/discharge cycle. Then, electrolytes are circulated back to their storage tanks. ${ }^{13,17}$ So far, transition metal-based redox flow batteries (such as vanadium, iron, and chromium) have found some commercial success. However, their widespread adoption has been limited mainly due to high production cost, toxicity, and cell component corrosion associated with the use of transition metal salts ${ }^{18,19}$. Therefore, redox flow batteries containing organic redox-active species are being heavily investigated due to their low production cost, access to a massive space of electroactive compounds, and low environmental impact. ${ }^{19,20}$ Many organic compounds such as quinones, viologens, flavins, thiazines, imides, and their derivatives have been investigated for redox-active species in both aqueous and non-aqueous RFBs. ${ }^{18,21,22}$ However, non-aqueous RFBs offer large operating voltage. ${ }^{21}$ Recently, phenazine derivatives have been shown as promising redox-active candidates in non-aqueous RFBs having high voltage and density. Therefore, 
phenazine derivatives are currently being investigated as candidates for novel redox-active

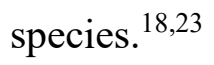

These investigations remain primarily experimental. Unfortunately, the vast chemical space offered by organic compounds cannot be explored using experimental procedures. Quantum mechanical DFT computations have been used heavily in chemistry research due to high accuracy but are very slow and cannot screen millions of molecules in a reasonable amount of time. Therefore, a fast and reliable method to screen millions of compounds without compromising accuracy is required. In this regard, machine-learning algorithms have shown excellent predictive accuracies along with short development and prediction times. ${ }^{24-28}$ Therefore, machine learning models have been used extensively to screen millions of molecules in materials science and drug discovery. ${ }^{29-33}$ Machine learning models generally require a large amount of data for accurate predictions. When the quantity of data is limited, feature engineering is employed to generate the most informative features. These features are expected to capture the appropriate molecular information necessary to predict the target variable. Feature engineering requires domain knowledge, relying on having access to experts. ${ }^{34-36}$ In small datasets, DFTbased or experimentally determined features have been used due to their high accuracy. However, some reports also explore simple features based on molecular structure. ${ }^{37-42}$

The goal of this study was to develop machine-learning models that predict redox potential in a short amount of time while maintaining high accuracy. Therefore, we did not compute any features from DFT calculations or experimental studies. The features used in this study were computed from molecular structures using readily available, easy-to-use Python libraries such as RDKit ${ }^{43}$ and DeepChem. ${ }^{44}$ These libraries have been used in other studies as well. ${ }^{45-48}$ Previous studies to predict redox potential using machine-learning investigate only a small number of non- 
linear models. ${ }^{49-53}$ Furthermore, none of the previous studies use easily computable features from RDKit or DeepChem libraries. This study investigates twenty different linear and non-linear machine-learning models to predict the redox potential of phenazine derivatives in DME (dimethoxyethane) solvent. Linear models are generally faster to train but may not capture complex relationships between features and target variables, whereas non-linear models are capable of capturing these complex relationships but may require a considerable amount of training time. A total of 3510 features containing 2D, 3D, and molecular fingerprints were generated using RDKit, and DeepChem. Models were trained on four feature sets described in Table 1 to obtain high prediction accuracy. Moreover, to understand which feature set had the best prediction accuracy, a detailed analysis of model performance was carried out using the "pipeline" developed in this work (described in the materials and methods section). The pipeline was developed to make training and evaluation easy, consistent, and automatic for all models. It combines different model training and evaluation steps into a single, convenient sub-routine. Then, the feature importance analysis was performed to identify the most important features in each feature set. Subsequent to that, model performance was analyzed on small subsets of the most important features to reduce training and inference time for large datasets. Then, the promising redox-active candidates were identified using the predictions from best-performing models. Also, the prediction accuracy across different functional groups was analyzed. Finally, the sources of errors were identified.

We believe that the methods used in this work are easily adaptable, and the results obtained in this study would help accelerate the discovery of novel redox-active species for energy storage applications. 


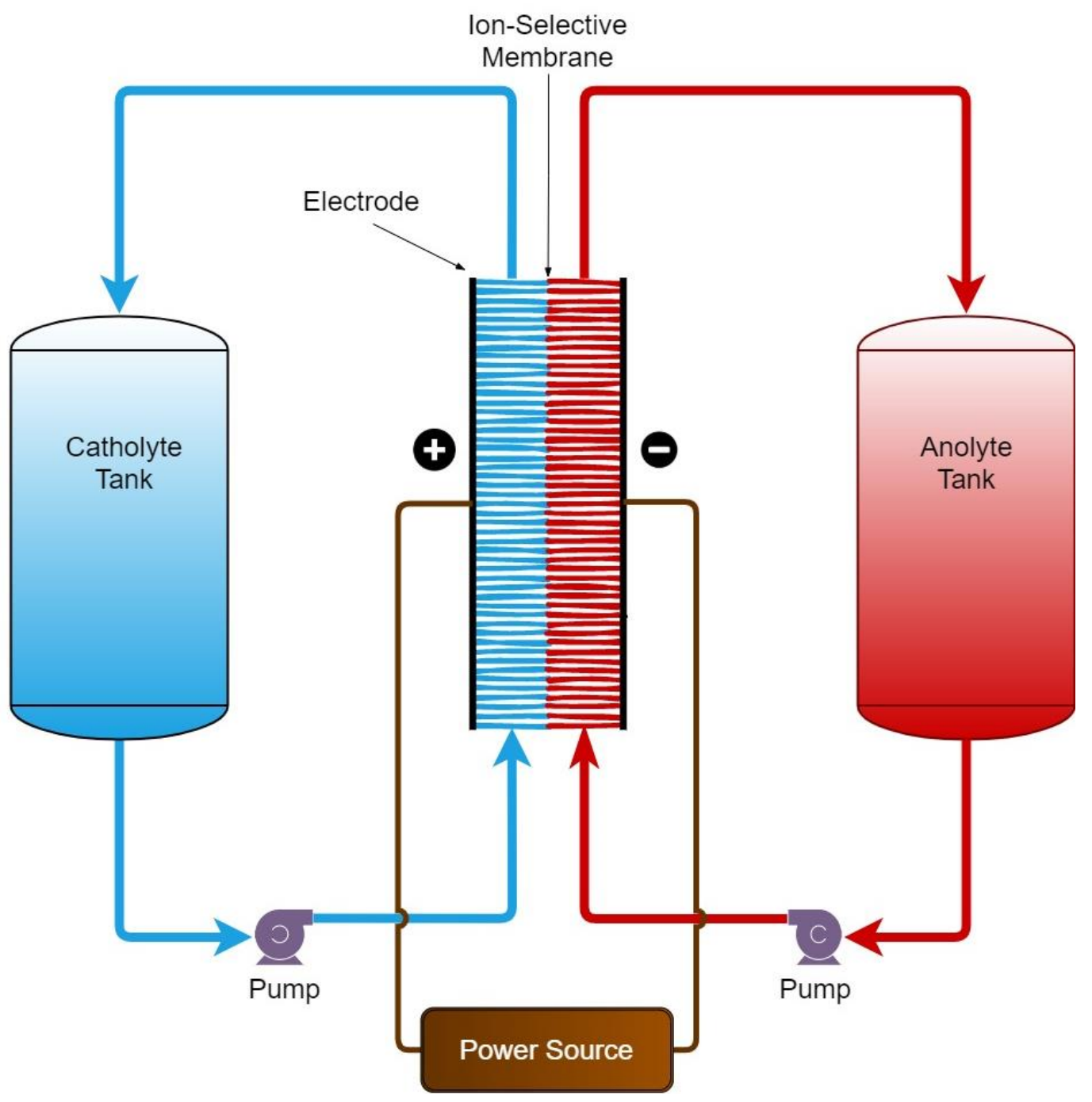

Figure 1. Schematic diagram of a typical redox flow battery 


\section{MATERIALS AND METHODS}

\subsection{Data set}

Data used in this study was obtained from the work reported by Mavrandonakis and coworkers. ${ }^{18}$ The redox potentials of 189 phenazine derivatives in DME were provided in that work. These potentials were computed using DFT. Phenazine derivatives contain twenty unique electron-withdrawing and donating functional groups $\left(-\mathrm{N}\left(\mathrm{CH}_{3}\right)_{2},-\mathrm{NH}_{2},-\mathrm{OH},-\mathrm{OCH}_{3}\right.$, $\mathrm{P}\left(\mathrm{CH}_{3}\right)_{2},-\mathrm{SCH}_{3},-\mathrm{SH},-\mathrm{CH}_{3},-\mathrm{C}_{6} \mathrm{H}_{5},-\mathrm{CH}=\mathrm{CH}_{2},-\mathrm{F},-\mathrm{Cl},-\mathrm{CHO},-\mathrm{COCH}_{3},-\mathrm{CONH}_{2},-$ $\mathrm{COOCH}_{3},-\mathrm{COOH},-\mathrm{CF}_{3},-\mathrm{CN}$ and $-\mathrm{NO}_{2}$ ). Optimized $3 \mathrm{D}$ structures of derivatives in neural and in anionic states were also provided. Only neutral structures were used for the feature computation. However, not all compounds were supplied with their neutral structure. Therefore, compounds missing neutral structures were removed. Thus, we ended up with 185 compounds in the final dataset. Next, 3510 different types of features were generated using RDKit and DeepChem, libraries as described below. Finally, the whole dataset was shuffled and split randomly into a train-set and a test-set, in a 7:3 ratio. This resulted in 129 samples in the train-set and 56 samples in the test-set.

\subsection{Feature Generation}

For each compound, three types of features were generated: (i) 2D, (ii) 3D, and (iii) molecular fingerprints. A list of all features used in this study is given in Table S1. Ten 2D features were generated from the raw data (features with the word 'basic' in the suffix). The rest of the 2D features were computed using RDKit. ${ }^{43}$ All 3D features were computed using RDKit. Molecular fingerprints were computed using RDKit and DeepChem ${ }^{44}$ libraries. These features were 
grouped into four sets, as shown in Table 1. Molecular fingerprints and some of the 3D features were 1D vectors. In this study, we considered each component of the vectorial feature as an independent feature. Therefore, a small number of unique 3D and Molecular Fingerprint features resulted in a large number of final features. Features having a "NaN" (Not a Number) value for any compound were removed. Also, features having identical values for all compounds were removed, as they did not contain any useful information. All 2D and 3D features computed from the RDKit library were scaled using the 'StandardScaler' class of the Scikit-learn library ${ }^{54}$, which removes mean and scales each feature to unit variance.

Table 1. Feature sets

\begin{tabular}{|l|l|l|l|}
\hline $\begin{array}{l}\text { Sr. } \\
\text { No }\end{array}$ & $\begin{array}{l}\text { Feature } \\
\text { Set }\end{array}$ & Description & $\begin{array}{l}\text { Number of } \\
\text { Features }\end{array}$ \\
\hline 1 & $2 \mathrm{~d}+3 \mathrm{~d}+\mathrm{fp}$ & $\begin{array}{l}\text { Contains 2D and 3D features computed using raw } \\
\text { data and RDKit. Also contains Molecular } \\
\text { Fingerprints computed from RDKit and DeepChem }\end{array}$ & 3510 \\
\hline 2 & $2 \mathrm{~d}$ & $\begin{array}{l}\text { Contains only 2D features computed using the raw } \\
\text { data and RDKit }\end{array}$ & 151 \\
\hline 3 & $3 \mathrm{~d}$ & Contains only 3D features computed using RDKit & 869 \\
\hline 4 & $\mathrm{fp}$ & $\begin{array}{l}\text { Contains Molecular Fingerprints computed using } \\
\text { DeepChem and RDKit }\end{array}$ & 2490 \\
\hline
\end{tabular}

\subsection{Machine-learning Models}

Twenty linear and non-linear machine-learning models were investigated in this study. Machine-learning models were implemented with the Scikit-learn Python library. ${ }^{54}$ A list of all models is given in Table 2. 
Table 2. Model list

\begin{tabular}{|c|c|c|c|}
\hline $\begin{array}{l}\text { Sr. } \\
\text { No. }\end{array}$ & Model Name & Alias & Model Type \\
\hline 1 & Linear Regression & linear_reg & Linear \\
\hline 2 & Ridge Regression & ridge & Linear \\
\hline 3 & Lasso & lasso & Linear \\
\hline 4 & Elastic-Net & elastic_net & Linear \\
\hline 5 & LARS Lasso & lasso_lars & Linear \\
\hline 6 & Orthogonal Matching Pursuit & omp & Linear \\
\hline 7 & Bayesian Ridge Regression & bayesian_ridge & Linear \\
\hline 8 & $\begin{array}{l}\text { Automatic Relevance Determination } \\
\text { Regression }\end{array}$ & ARDR & Linear \\
\hline 9 & Passive Aggressive & $\mathrm{PA}$ & Linear \\
\hline 10 & Huber Regression & huber & Linear \\
\hline 11 & Kernel ridge Regression & kernel_ridge & Non-Linear \\
\hline 12 & Support Vector Machines & SVR & Non-Linear \\
\hline 13 & Gaussian Processes Regression & gaussian_process & Non-Linear \\
\hline 14 & Decision Trees & decision_tree & Non-Linear \\
\hline 15 & Bagging meta-estimator & bagging & Non-Linear \\
\hline 16 & Random Forest & random_forest & Non-Linear \\
\hline 17 & AdaBoost & ada_boost & Non-Linear \\
\hline 18 & Gradient Boosting Regression & gradient_boosting_reg & Non-Linear \\
\hline 19 & Artificial Neural Network & neural_network & Non-Linear \\
\hline 20 & Nearest Neighbors Regression & knn_reg & Non-Linear \\
\hline
\end{tabular}




\subsection{Hyper-Parameter Tuning}

Hyper-Parameter tuning was performed for all models using the 'GridSearchCV' class of the Scikit-learn library. 'GridSearchCV' performs a systematic search over a grid of parameters to identify the best set of parameters using cross-validation. 10-fold cross-validation with mean squared error (MSE) loss was used in this study.

\subsection{Evaluation Metrics}

The following metrics were used to evaluate the model performance. In the formulas below, $N$ denotes the number of data points, $\widehat{y}_{l}$ denotes the predicted value of $i$-th sample and the $y_{i}$ denotes the corresponding true value.

1. Coefficient of Determination $\left(\mathrm{R}^{2}\right)$ :

$$
\begin{gathered}
R^{2}=1-\frac{\sum_{i=1}^{N}\left(y_{i}-\widehat{y}_{l}\right)^{2}}{\sum_{i=1}^{N}\left(y_{i}-\bar{y}\right)^{2}} \\
\text { where, } \quad \bar{y}=\frac{\sum_{i=1}^{N} y_{i}}{N}
\end{gathered}
$$

2. Mean Squared Error (MSE):

3. Mean Absolute Error (MAE):

$$
M S E=\frac{\sum_{i=1}^{N}\left(y_{i}-\hat{y}\right)^{2}}{N}
$$

$$
M A E=\frac{\sum_{i=1}^{N}\left|y_{i}-\widehat{y}_{\imath}\right|}{N}
$$

4. Mean Absolute Percentage Error (MAPE):

$$
M A P E=100 * \frac{\sum_{i=1}^{N} \frac{\left|y_{i}-\hat{y}_{l}\right|}{\left\lceil y_{i} \mid\right.}}{N}
$$


MSE was used as an internal evaluation metric in all cross-validation procedures. Other metrics were used to get more interpretable results. The use of terms 'Accuracy' and 'Performance' in this report is contextual and refers to one or more metrics defined above.

\subsection{MSE and MAE Threshold}

To understand whether the model was learning or not, we determined an approximate upper bound on MSE and MAE for the train and test set. It is expected that MSE and MAE would stay below this threshold if learning were successful. It was observed that when the training fails, the model predicts a constant value (i.e., the mean of the training data). Therefore, the threshold value for MSE and MAE was determined using the mean value of training data. The threshold values are shown in Table 3.

Table 3. Threshold values

\begin{tabular}{|l|l|l|l|}
\hline Sr. No. & Metric & Train-Set Threshold & Test-Set Threshold \\
\hline 1 & MSE & 0.47 & 0.44 \\
\hline 2 & MAE & 0.6 & 0.56 \\
\hline
\end{tabular}

\section{7 k-Fold Cross-Validation}

In a typical $\mathrm{k}$-fold cross-validation procedure, the train-set is split into $\mathrm{k}$ sets of approximately equal size. Then, the model is trained on k-1 sets, leaving one set as a test-set. Then, the performance of the trained model is evaluated on the left-out test-set. This procedure is repeated for every fold, and the average performance is reported. As every data point in the training set is evaluated as if it belongs to the test-set, the performance obtained from cross-validation is considered a reasonable estimate of out-of-sample performance. k-fold cross-validation gives 
robust out-of-sample performance for the model. It is a crucial evaluation technique, especially when the dataset size is very small, and it becomes impractical to partition data into three sets (i.e., train, validation, test). 10-fold cross-validation with MSE loss was used in this study.

\subsection{Feature Importance Score}

Feature importance scores were computed using the optimized version of Random Forest, AdaBoost, and Gradient Boosting Regression trained on all features from the corresponding feature set.

\subsection{Pipeline}

To assess the model performance, we developed a pipeline that combines all training and evaluation components into a single procedure. Given the train and test sets as inputs, the pipeline first performs hyperparameter-tuning for all models, then evaluates the performance of optimized models on $\mathrm{CV}$, train, and test sets, and finally combines necessary results from each step in a single dataframe. Pipeline makes the training and evaluation easy, consistent and automatic for all models across different scenarios. A pictorial representation of the pipeline is shown in Figure 2. Different steps in the pipeline are described below:

i. Input: First, training and test data are provided as inputs.

ii. Hyper-Parameter Tuning: In this step, optimized parameters of all twenty models are determined using the train-set, as described earlier in this section.

iii. 10-Fold Cross-Validation: In this step, the cross-validation performance of optimized models is evaluated using 10 -fold cross-validation on the train-set. Three metrics (i.e., $\mathrm{R}^{2}$, MSE, and MAE) are recorded during the cross-validation for all models. 
iv. Train and Test set Performance: In this step, the performance of all optimized models is evaluated on the train and test set. Three metrics (i.e., $\mathrm{R}^{2}$, MSE, and MAE) are recorded during the evaluation for all models.

v. Output: In this step, results from steps ii-iv are combined into one dataframe containing the best set of parameters, 10 -fold cross-validation performance, train and test set performance of all models.
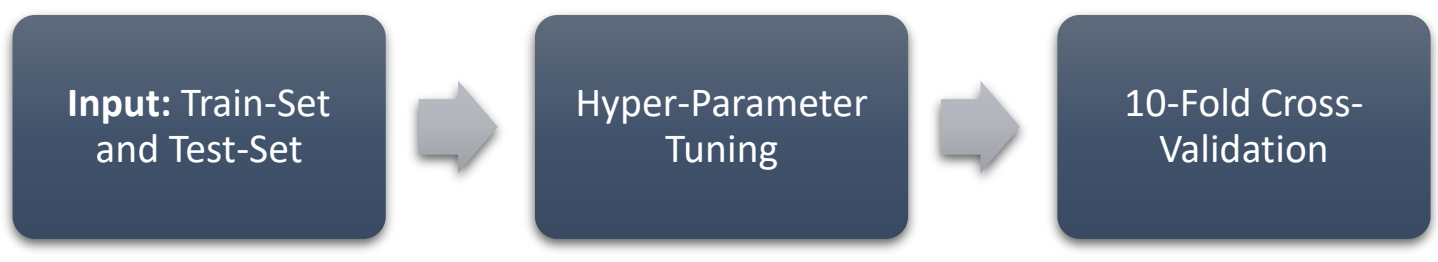

\section{Output: Optimized \\ Models, Evaluation \\ Metrics etc.}

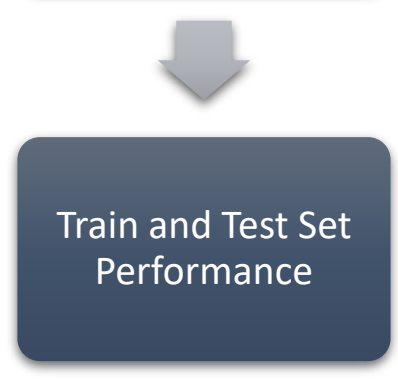

Figure 2. Pictorial representation of the training and evaluation pipeline. 


\section{RESULTS AND DISCUSSION}

\subsection{Analysis of the Best-Performing Models}
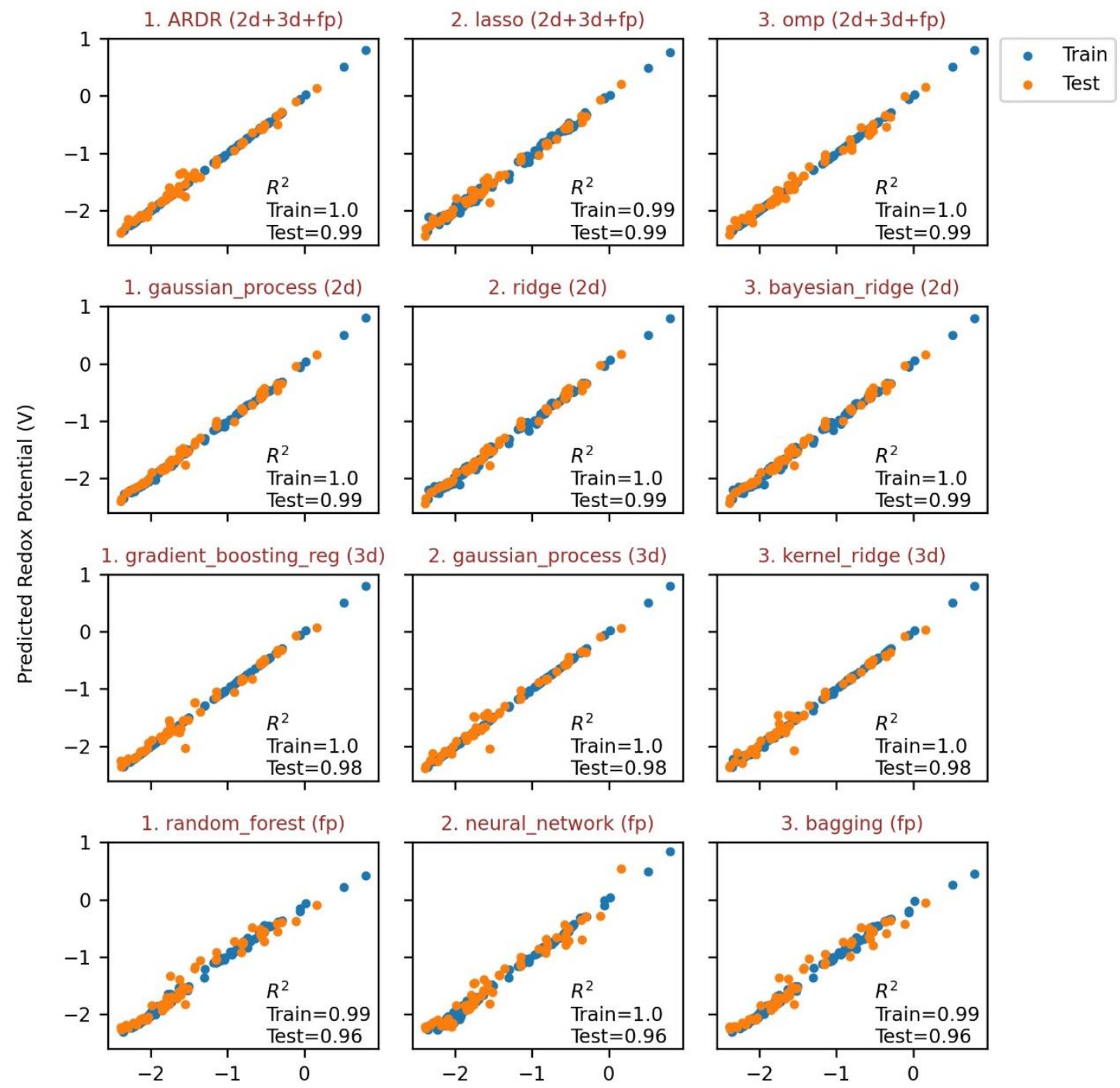

True Redox Potential (V)

Figure 3. Machine-learning prediction of redox potential vs. true redox potential of the three best-performing models in each feature sets. The title of each plot indicates model name, its rank, and the corresponding feature set used for training in brackets. 
For accurate prediction of the redox potential of phenazine derivatives, we employed twenty different linear and non-linear machine-learning models, which are listed in Table 2. The whole dataset was shuffled and split randomly into train and test sets in a 7:3 ratio. The size of the train and test set was 129 and 56, respectively. Even though models were trained on a relatively small dataset, they achieved excellent performance on the unseen data (i.e., test-set). Figure 3 shows the redox potentials predicted by models on the y-axis and the corresponding true value of redox potentials on the $\mathrm{x}$-axis. It can be seen that the majority of models achieved an $\mathrm{R}^{2}$ value of 0.99 on the test-set ( $\mathrm{R}^{2}$ values in the plots were rounded to two decimal places for clarity). Table 4 shows the fifteen best-performing models obtained in this study along with their $\mathrm{R}^{2}$, MSE, and MAE values on cross-validation (CV), train-set, and test-set. All top twenty models not only had an outstanding performance on the train-set but also on the test-set (i.e., $\mathrm{R}^{2}>0.98$, MSE $<0.008$ $\mathrm{V}^{2}$ and $\mathrm{MAE}<0.07 \mathrm{~V}$ ). 


\begin{tabular}{|c|c|c|c|c|c|c|c|c|c|c|c|c|c|c|c|}
\hline$\vec{u}$ & $\vec{D}$ & $\varpi$ & $\bar{N}$ & 二 & $\overline{0}$ & 6 & $\infty$ & $\checkmark$ & $a$ & $u$ & $\triangle$ & $\omega$ & $N$ & - & $\begin{array}{l}\text { ટ } \\
\text { ह્र }\end{array}$ \\
\hline 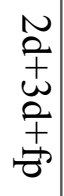 & $\begin{array}{l}N \\
\stackrel{N}{+} \\
+ \\
\stackrel{2}{+} \\
+ \\
\vec{\sigma}\end{array}$ & 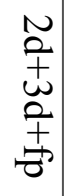 & 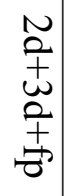 & $\stackrel{N}{2}$ & 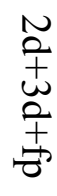 & 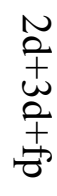 & $\begin{array}{l}N \\
\stackrel{0}{+} \\
\dot{W} \\
\stackrel{0}{+} \\
\vec{\sigma}\end{array}$ & $\stackrel{N}{2}$ & $\stackrel{N}{2}$ & $\stackrel{N}{2}$ & $\stackrel{N}{\varrho}$ & $\stackrel{N}{2}$ & $\tilde{\Omega}$ & $\tilde{\Omega}$ & 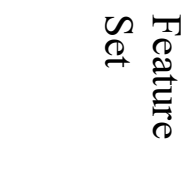 \\
\hline 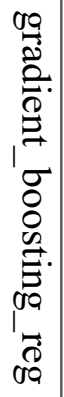 & 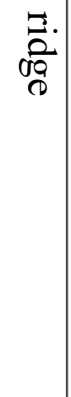 & 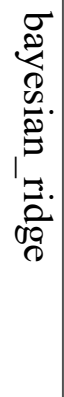 & 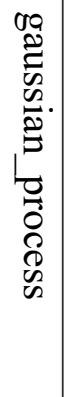 & $\begin{array}{l}\overline{0} \\
\text { Dे } \\
0\end{array}$ & $\stackrel{\varrho}{\Xi}$ & $\begin{array}{l}\overline{0} \\
\overline{0} \\
\text { d }\end{array}$ & $\underset{\nabla}{\vec{\theta}}$ & $\overbrace{0}^{D}$ & $\stackrel{O}{\Xi}$ & 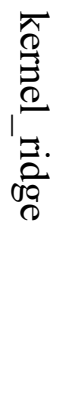 & 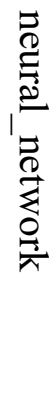 & 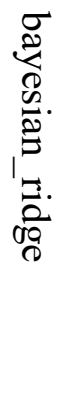 & 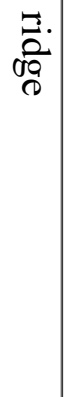 & 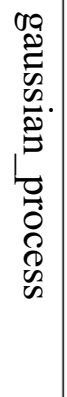 & 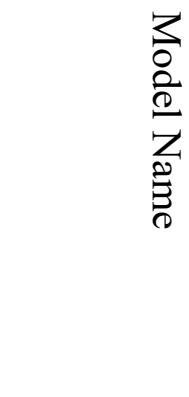 \\
\hline $\begin{array}{l}\stackrel{0}{0} \\
\underset{1}{\omega}\end{array}$ & $\begin{array}{l}0 \\
\dot{0} \\
\infty \\
\cup_{1}\end{array}$ & \begin{tabular}{l}
0 \\
$\dot{0}$ \\
$\infty$ \\
\hdashline
\end{tabular} & $\begin{array}{l}0 \\
0 \\
\infty \\
+0\end{array}$ & $\begin{array}{l}\text { o } \\
\stackrel{\sigma}{\alpha} \\
\infty\end{array}$ & 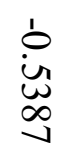 & $\begin{array}{l}0 \\
\dot{0} \\
\infty \\
\tilde{N}\end{array}$ & $\begin{array}{l}0 \\
\dot{u} \\
\text { ư }\end{array}$ & 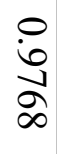 & $\begin{array}{l}0 \\
i 0 \\
8\end{array}$ & $\begin{array}{l}0 \\
\dot{b} \\
w \\
\infty\end{array}$ & $\begin{array}{l}0 \\
\dot{0} \\
\stackrel{1}{+} \\
+\end{array}$ & $\begin{array}{l}0 \\
\dot{0} \\
\text { ư } \\
\text { u }\end{array}$ & $\begin{array}{l}0 \\
\dot{0} \\
a \\
\vdots\end{array}$ & $\begin{array}{l}0 \\
\dot{0} \\
w \\
\infty\end{array}$ & $\overbrace{}^{\pi}{ }_{N}$ \\
\hline $\begin{array}{l}0 \\
\stackrel{0}{0} \\
\stackrel{\vec{D}}{ \pm}\end{array}$ & $\begin{array}{l}\circ \\
\stackrel{8}{8} \\
\dot{\omega}\end{array}$ & $\begin{array}{l}\circ \\
\dot{8} \\
\stackrel{0}{1} \\
\text { ur }\end{array}$ & $\begin{array}{l}\stackrel{0}{8} \\
\dot{0} \\
\text { ஸ }\end{array}$ & $\begin{array}{l}0 \\
\dot{8} \\
\stackrel{1}{\infty}\end{array}$ & $\begin{array}{l}0 \\
i \omega \\
\omega \\
\omega\end{array}$ & $\begin{array}{l}\circ \\
\stackrel{8}{8} \\
\stackrel{1}{1}\end{array}$ & 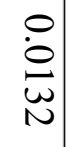 & $\begin{array}{l}0 \\
\dot{8} \\
\stackrel{1}{1}\end{array}$ & $\begin{array}{l}0 \\
0 \\
\text { प } \\
\frac{1}{a}\end{array}$ & $\begin{array}{l}0 \\
\dot{8} \\
\text { ป }\end{array}$ & $\begin{array}{l}\stackrel{0}{0} \\
\stackrel{0}{\circ}\end{array}$ & $\begin{array}{l}0 \\
\stackrel{0}{0} \\
\text { N }\end{array}$ & $\begin{array}{l}0 \\
\dot{8} \\
8 \\
0\end{array}$ & $\begin{array}{l}\stackrel{0}{8} \\
\stackrel{0}{0} \\
\infty\end{array}$ & 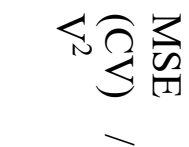 \\
\hline $\begin{array}{l}0 \\
\stackrel{8}{9} \\
\dot{\omega}\end{array}$ & $\begin{array}{l}0 \\
\stackrel{1}{b} \\
0\end{array}$ & $\begin{array}{l}0 \\
\dot{0} \\
\text { प̆ } \\
0\end{array}$ & $\begin{array}{l}0 \\
\dot{0} \\
\text { प̆ } \\
0\end{array}$ & $\begin{array}{l}0 \\
\dot{2} \\
\infty\end{array}$ & $\begin{array}{l}\stackrel{0}{N} \\
\stackrel{\omega}{v}\end{array}$ & $\begin{array}{l}0 \\
\dot{0} \\
\text { U్ } \\
\omega\end{array}$ & $\begin{array}{l}0 \\
\stackrel{\circ}{8} \\
8\end{array}$ & $\begin{array}{l}\stackrel{0}{0} \\
\text { ஸे } \\
\text { N }\end{array}$ & $\begin{array}{l}\stackrel{0}{0} \\
\stackrel{1}{0} \\
\infty\end{array}$ & 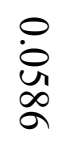 & $\begin{array}{l}\stackrel{0}{0} \\
8 \\
\frac{1}{6}\end{array}$ & $\begin{array}{l}\stackrel{0}{0} \\
\text { U্ } \\
\text { v }\end{array}$ & $\begin{array}{l}0 \\
\dot{0} \\
\text { ب̂ } \\
\pm\end{array}$ & $\begin{array}{l}0 \\
\dot{0} \\
\text { Й } \\
\text { ل1 }\end{array}$ & $<\overbrace{S}^{2} \underset{\pi}{3}$ \\
\hline$\dot{8}$ & $\begin{array}{l}0 \\
6 \\
0 \\
0\end{array}$ & $\begin{array}{l}0 \\
i 0 \\
0 \\
0 \\
\omega\end{array}$ & $\dot{8}$ & $\begin{array}{l}0 \\
\dot{0} \\
0\end{array}$ & ஜ & $\begin{array}{l}0 \\
\text { ర্ } \\
\text { w }\end{array}$ & 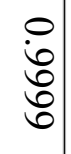 & $\begin{array}{l}0 \\
\text { ర0 } \\
\text { पू }\end{array}$ & $\begin{array}{l}0 \\
\dot{0} \\
0 \\
0\end{array}$ & $\begin{array}{l}0 \\
\dot{0} \\
\stackrel{0}{ \pm}\end{array}$ & $\begin{array}{l}0 \\
\dot{0} \\
\infty \\
\infty\end{array}$ & $\begin{array}{l}0 \\
i 0 \\
0 \\
\circ \\
1\end{array}$ & $\begin{array}{l}0 \\
\dot{0} \\
8 \\
8\end{array}$ & $\begin{array}{l}0 \\
\dot{0} \\
0 \\
0\end{array}$ & 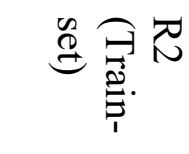 \\
\hline $\begin{array}{l}0 \\
8 \\
8 \\
8\end{array}$ & \begin{tabular}{l}
0 \\
8 \\
8 \\
\hdashline
\end{tabular} & $\begin{array}{l}0 \\
8 \\
8 \\
0\end{array}$ & $\begin{array}{l}0 \\
8 \\
8 \\
8\end{array}$ & $\begin{array}{l}\stackrel{0}{8} \\
\stackrel{8}{a}\end{array}$ & $\begin{array}{l}\circ \\
8 \\
8\end{array}$ & $\begin{array}{l}\stackrel{0}{8} \\
\stackrel{8}{0}\end{array}$ & $\begin{array}{l}\circ \\
8 \\
8 \\
8\end{array}$ & $\begin{array}{l}\stackrel{0}{0} \\
\stackrel{8}{0} \\
\text { O }\end{array}$ & $\begin{array}{l}\stackrel{0}{8} \\
\stackrel{8}{0} \\
\infty\end{array}$ & $\begin{array}{l}0 \\
8 \\
0 \\
\text { U }\end{array}$ & $\begin{array}{l}0 \\
\dot{8} \\
8 \\
8\end{array}$ & $\begin{array}{l}\stackrel{0}{8} \\
\stackrel{0}{V}\end{array}$ & $\begin{array}{l}0 \\
\dot{8} \\
0 \\
0\end{array}$ & $\begin{array}{l}0 \\
\dot{8} \\
8 \\
+\end{array}$ & 〈丶 \\
\hline $\begin{array}{l}0 \\
\dot{8} \\
8 \\
0\end{array}$ & $\begin{array}{l}0 \\
\stackrel{0}{+} \\
\stackrel{P}{\perp}\end{array}$ & $\begin{array}{l}0 \\
\stackrel{0}{\Xi} \\
\stackrel{\square}{+}\end{array}$ & $\begin{array}{l}0 \\
\dot{8} \\
\text { N }\end{array}$ & $\begin{array}{l}0 \\
\dot{0} \\
\text { Ŭ } \\
0\end{array}$ & $\begin{array}{l}0 \\
8 \\
8\end{array}$ & $\begin{array}{l}\dot{0} \\
\stackrel{+}{1} \\
0\end{array}$ & $\begin{array}{l}0 \\
\dot{8} \\
\stackrel{0}{N}\end{array}$ & $\begin{array}{l}.0 \\
\dot{0} \\
\dot{w}\end{array}$ & $\begin{array}{l}0 \\
\dot{\infty} \\
\infty \\
0\end{array}$ & $\begin{array}{l}0 \\
\dot{\omega} \\
\stackrel{N}{N}\end{array}$ & $\begin{array}{l}\stackrel{0}{0} \\
\stackrel{\omega}{\omega}\end{array}$ & $\begin{array}{l}\stackrel{0}{0} \\
\text { N } \\
\text { N }\end{array}$ & 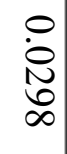 & $\begin{array}{l}0 \\
0 \\
\omega \\
\sigma\end{array}$ & 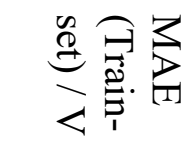 \\
\hline $\begin{array}{l}0 \\
0 \\
\infty \\
+0\end{array}$ & $\begin{array}{l}0 \\
i 0 \\
\infty \\
\mathbb{0}\end{array}$ & $\begin{array}{l}0 \\
\dot{0} \\
\infty \\
\omega \\
\omega\end{array}$ & $\begin{array}{l}0 \\
0 \\
\infty \\
\text { un }\end{array}$ & $\begin{array}{l}0 \\
\dot{0} \\
\infty \\
y\end{array}$ & $\begin{array}{l}0 \\
0 \\
\infty \\
0\end{array}$ & $\begin{array}{l}0 \\
0 \\
\infty \\
\infty\end{array}$ & $\begin{array}{l}0 \\
\dot{0} \\
\infty \\
w\end{array}$ & $\begin{array}{l}0 \\
\dot{0} \\
\infty \\
\mathbb{1}\end{array}$ & $\begin{array}{l}0 \\
\dot{0} \\
\infty \\
a\end{array}$ & $\begin{array}{l}0 \\
i 0 \\
\infty \\
0 \\
0\end{array}$ & $\begin{array}{l}0 \\
i 0 \\
0 \\
0\end{array}$ & $\begin{array}{l}0 \\
\dot{0} \\
\text { U }\end{array}$ & $\begin{array}{l}0 \\
6 \\
0 \\
a\end{array}$ & $\begin{array}{l}\text { 웅 } \\
\text { N }\end{array}$ & 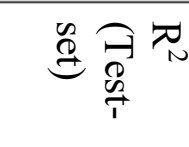 \\
\hline $\begin{array}{l}\circ \\
\dot{8} \\
\circ \\
2\end{array}$ & $\begin{array}{l}0 \\
8 \\
\check{\delta}\end{array}$ & $\begin{array}{l}0 \\
\dot{8} \\
\circ \\
+\end{array}$ & $\begin{array}{l}0 \\
\dot{8} \\
\circ \\
+\end{array}$ & $\begin{array}{l}\text { o } \\
\dot{8} \\
\text { 心 }\end{array}$ & $\begin{array}{l}\text { 영 } \\
\text { ᄋ }\end{array}$ & $\begin{array}{l}0 \\
\stackrel{8}{8} \\
\text { U }\end{array}$ & $\begin{array}{l}0 \\
\dot{8} \\
\text { யூ } \\
\sigma\end{array}$ & $\begin{array}{l}0 \\
8 \\
8 \\
\text { ư } \\
\text { ur }\end{array}$ & $\begin{array}{l}0 \\
8 \\
\dot{0} \\
\text { ur }\end{array}$ & $\begin{array}{l}0 \\
8 \\
\frac{1}{a}\end{array}$ & $\begin{array}{l}0 \\
8 \\
8 \\
0\end{array}$ & $\begin{array}{l}\stackrel{0}{8} \\
\dot{0}\end{array}$ & $\begin{array}{l}0 \\
\dot{8} \\
\text { U }\end{array}$ & $\begin{array}{l}0 \\
\dot{8} \\
\dot{w} \\
\text { u }\end{array}$ & 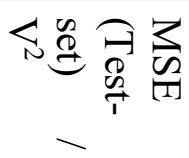 \\
\hline $\begin{array}{l}0 \\
\dot{0} \\
\text { पे } \\
\text { d }\end{array}$ & $\begin{array}{l}0 \\
\circ \\
\stackrel{+}{0} \\
+\end{array}$ & $\begin{array}{l}0 \\
\stackrel{+}{+} \\
\infty\end{array}$ & $\begin{array}{l}\stackrel{\circ}{+} \\
\stackrel{\infty}{二}\end{array}$ & $\begin{array}{l}0 \\
\dot{0} \\
\text { نे } \\
0\end{array}$ & 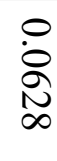 & $\begin{array}{l}0 \\
\dot{0} \\
\text { u }\end{array}$ & $\begin{array}{l}\circ \\
\stackrel{\circ}{\nexists} \\
\dot{\omega}\end{array}$ & $\begin{array}{l}0 \\
\dot{0} \\
\text { 11 } \\
0\end{array}$ & $\begin{array}{l}0 \\
\dot{0} \\
\text { ğ }\end{array}$ & $\begin{array}{l}\dot{0} \\
\dot{0} \\
\dot{0}\end{array}$ & $\begin{array}{l}\stackrel{0}{\stackrel{8}{+}} \\
\stackrel{+}{ \pm}\end{array}$ & $\begin{array}{l}\stackrel{0}{\stackrel{+}{N}} \\
\text { N }\end{array}$ & $\begin{array}{l}0 \\
\dot{1} \\
\text { 辛 }\end{array}$ & $\begin{array}{l}0 \\
\stackrel{1}{+} \\
\infty\end{array}$ & 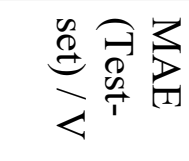 \\
\hline
\end{tabular}




\subsection{Assessment of Model Performance on Four Feature Sets}

The performance of machine-learning models depends on the type and the quality of features used for training. Therefore, it is important to identify the best set of features that achieve high prediction accuracy. Hence, we assessed model performance on four sets of features shown in Table 1. The goal here was to understand how different types of molecular features affected model performance. The list of all features is given in Table S1. The model performance on each feature set was assessed using the pipeline described in the materials and methods section. Fifteen best-performing models in Table 4 were obtained after assessing model performance independently on four feature sets. The 'Feature Set' column in Table 4 shows the corresponding feature set used for the training. Gaussian Processes Regression trained on 2D features achieved the highest prediction accuracy in this study. The negative value of $\mathrm{R}^{2}(\mathrm{CV})$ for the Orthogonal Matching Pursuit (omp) model in Table 4 suggests that it may not generalize well to the unseen data. A similar trend was observed for a few other linear models (Figure 4). Nine models, including the top seven models in Table 4, were trained on the ' $2 d$ ' feature set. The rest of the models in Table 4 were trained on the ' $2 d+3 d+f p$ ' feature set. We did not observe any model trained on either ' $3 d$ ' or ' $f p$ ' even in the top twenty best-performing models. The best-performing model in each feature set, along with their test-set performance (i.e., $\mathrm{R}^{2}$, MSE, and MAE), is shown in Table 5. Additionally, ten best-performing models in each feature set, along with their $\mathrm{CV}$, train-set, and test-set performance (i.e., $\mathrm{R}^{2}$, MSE, and MAE), are shown in Tables S2-S5. Similarly, the best-performing feature set for each model, along with their CV, train-set, and testset performance, is shown in Table S6. Observations from Table 4, Table 5 and Tables S2-S6 show that the prediction accuracy with respect to feature set follows the order: $2 d>2 d+3 d+f p>$ $3 d>f p$. Therefore, we conclude that 2D features are more informative than $3 \mathrm{D}$ and molecular 
fingerprint features to predict the redox potential of phenazine derivates in DME. Tables S2-S5 also show that linear models (e.g., ARDR, lasso, omp, ridge, bayesian_ridge) perform better than non-linear models on ' $2 d+3 d+f p$ ' and ' $2 d$ ' feature sets, whereas non-linear models (e.g., gradient_boosting_reg, gaussian_process, random_forest, neural_network) perform better than linear models on ' $2 d$ ', ' $3 d$ ' and ' $f p$ ' feature sets. This observation suggests that linear models should be preferred when the feature set consists of different features (i.e., 2D, 3D, and Molecular Fingerprints). Non-linear models should be preferred when the feature set contains either 3D or molecular fingerprint features. Any model could be used for 2D features. Linear models are generally faster than non-linear models due to their simple structure but are not preferred due to low accuracy. The results obtained here show that linear models could give accurate predictions compared to non-linear models in certain combinations of features $(2 d+3 d+f p$ ' feature set in this study). Utilizing linear models in these scenarios could significantly reduce the training and inference time.

Table 5. Test-set performance of the best-performing models in each feature set. Models were trained on all features from the corresponding feature set.

\begin{tabular}{|l|l|l|l|l|l|}
\hline $\begin{array}{l}\text { Sr. } \\
\text { No. }\end{array}$ & Featue Set & Model Name & $\begin{array}{l}\mathrm{R}^{2} \text { (Test- } \\
\text { set) }\end{array}$ & $\begin{array}{l}\text { MSE (Test- } \\
\text { set) / V }\end{array}$ & $\begin{array}{l}\text { MAE (Test- } \\
\text { set) / V }\end{array}$ \\
\hline 1 & $2 \mathrm{~d}+3 \mathrm{~d}+\mathrm{fp}$ & ARDR & 0.9873 & 0.0056 & 0.0473 \\
\hline 2 & $2 \mathrm{~d}$ & gaussian_process & 0.9921 & 0.0035 & 0.0428 \\
\hline 3 & $3 \mathrm{~d}$ & gradient_boosting_reg & 0.9788 & 0.0093 & 0.0573 \\
\hline 4 & $\mathrm{fp}$ & random_forest & 0.9583 & 0.0183 & 0.1012 \\
\hline
\end{tabular}




\subsection{Cross-Validation and Out-of-Sample Performance}

10-fold cross-validation (CV) performance (i.e., $\mathrm{R}^{2}$, MSE, and MAE) obtained from the pipeline is shown in Figure 4 for all twenty models. Cross-validation gives a reasonable estimate of out-of-sample performance (i.e., performance on unseen data). ' $2 d+3 d+f p$ ', ' $2 d$ ' and ' $3 d$ ' feature sets had the acceptable CV performance (i.e., MSE and MAE below their threshold value) on most models except for four linear models. The computation of threshold values for MSE and MAE is described in the materials and methods section. These four linear models (i.e., linear_reg, omp, PA, huber) had negative $\mathrm{R}^{2}$ value, high MSE, and high MAE (i.e., close to threshold) for at least one feature set. ' $f p$ ' feature set had the worst CV performance on all models. Three linear models (i.e., omp, $P A$, huber) had poor $\mathrm{CV}$ performance on the ' $3 d$ ' feature set. Performance on test-set (i.e., $\mathrm{R}^{2}$, MSE, and MAE) obtained from the pipeline is shown in Figure 5 for all twenty models. As models never see the test-set, this gives an even better estimate of out-of-sample performance than cross-validation. All feature sets had an acceptable test-set performance on all models except Linear Regression. Linear Regression had a poor testset performance on ' $2 d$ ' and ' $f p$ ' feature sets. Furthermore, the averaged train and test performance (i.e., $\mathrm{R}^{2}$, MSE, and MAE values were averaged over all models) for each feature set are shown in Table 6. Values of linear Regression were not considered in the average due to very high errors. In Table $6, ' 2 d+3 d+f p$ ' and ' $f p$ ' feature sets had better train-set performance and poorer test-set performance in comparison to the ' $2 d$ ' feature set. This indicates that $2 \mathrm{D}$ features are better at generalizing to unseen data than $3 \mathrm{D}$ and molecular fingerprint features. Trend analysis of Figure 4, Figure 5, and Table 6 revealed the previously observed order of feature set performance, $2 d>2 d+3 d+f p>3 d>f p$. Plots similar to Figure 5 for the train-set are shown in Figure S1. 
Table 6. Train and test set performance of four feature sets averaged over all models except Linear Regression. Models were trained on all features from the corresponding feature set.

\begin{tabular}{|l|l|l|l|l|l|l|l|}
\hline Sr. No. & $\begin{array}{l}\text { Feature } \\
\text { Set }\end{array}$ & $\begin{array}{l}\mathrm{R}^{2} \\
(\text { Train- } \\
\text { set })\end{array}$ & $\begin{array}{l}\text { MSE } \\
(\text { Train- } \\
\text { set) } / \mathrm{V}^{2}\end{array}$ & $\begin{array}{l}\text { MAE } \\
(\text { Train- } \\
\text { set) } / \mathrm{V}\end{array}$ & $\begin{array}{l}\mathrm{R}^{2} \\
(\text { Test- } \\
\text { set })\end{array}$ & $\begin{array}{l}\text { MSE } \\
(\text { Test- } \\
\text { set) } / \mathrm{V}^{2}\end{array}$ & $\begin{array}{l}\text { MAE } \\
(\text { Test- } \\
\text { set) } / \mathrm{V}\end{array}$ \\
\hline 1 & $2 \mathrm{~d}+3 \mathrm{~d}+\mathrm{fp}$ & 0.9926 & 0.0035 & 0.0301 & 0.9718 & 0.0124 & 0.0733 \\
\hline 2 & $2 \mathrm{~d}$ & 0.9876 & 0.0058 & 0.0426 & 0.9729 & 0.0119 & 0.0734 \\
\hline 3 & $3 \mathrm{~d}$ & 0.9917 & 0.0039 & 0.0335 & 0.9535 & 0.0204 & 0.0899 \\
\hline 4 & $\mathrm{fp}$ & 0.9718 & 0.0132 & 0.0506 & 0.9028 & 0.0427 & 0.1511 \\
\hline
\end{tabular}



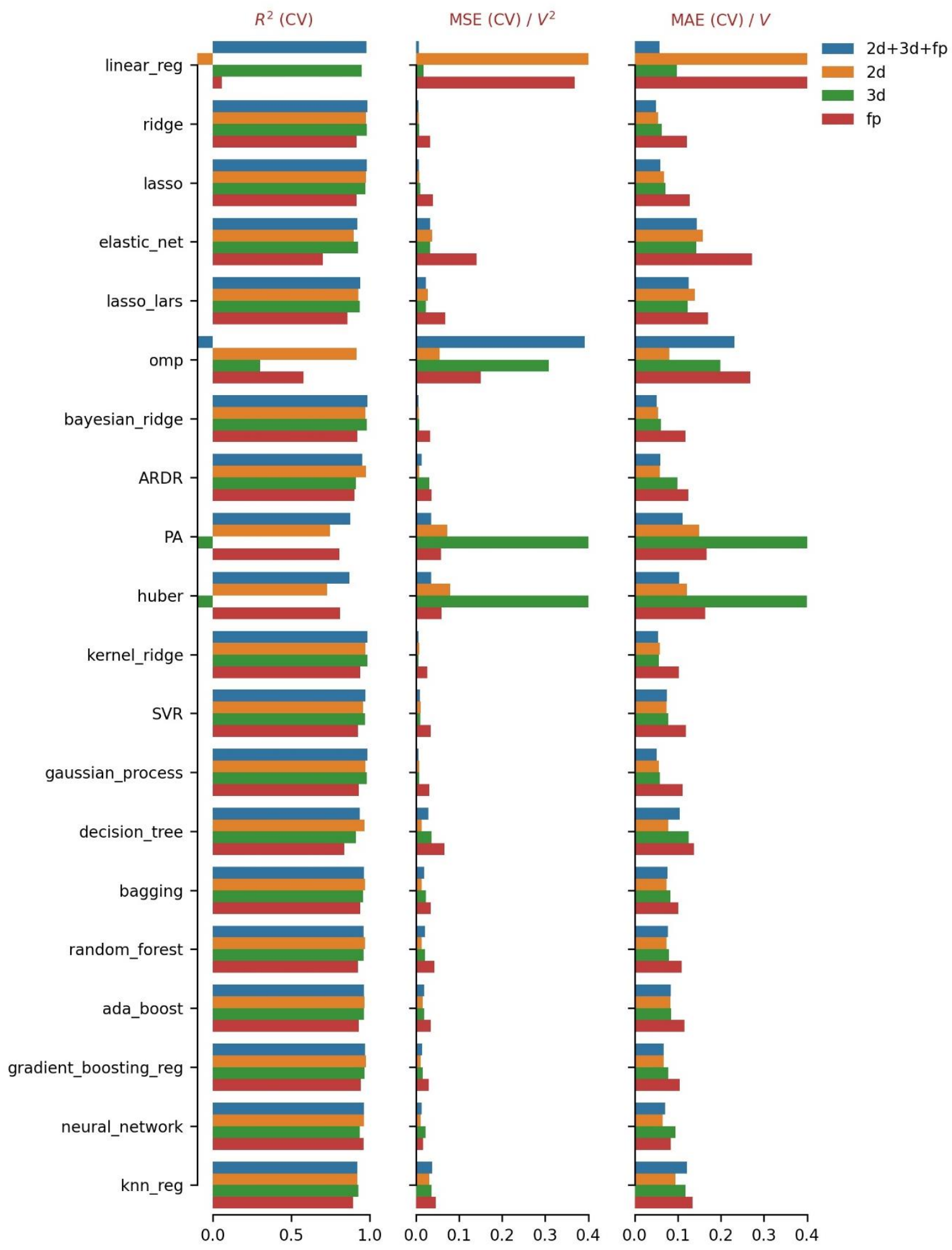

Figure 4. 10-Fold cross-validation performance of twenty models. Models were trained on all features from the corresponding feature set. 


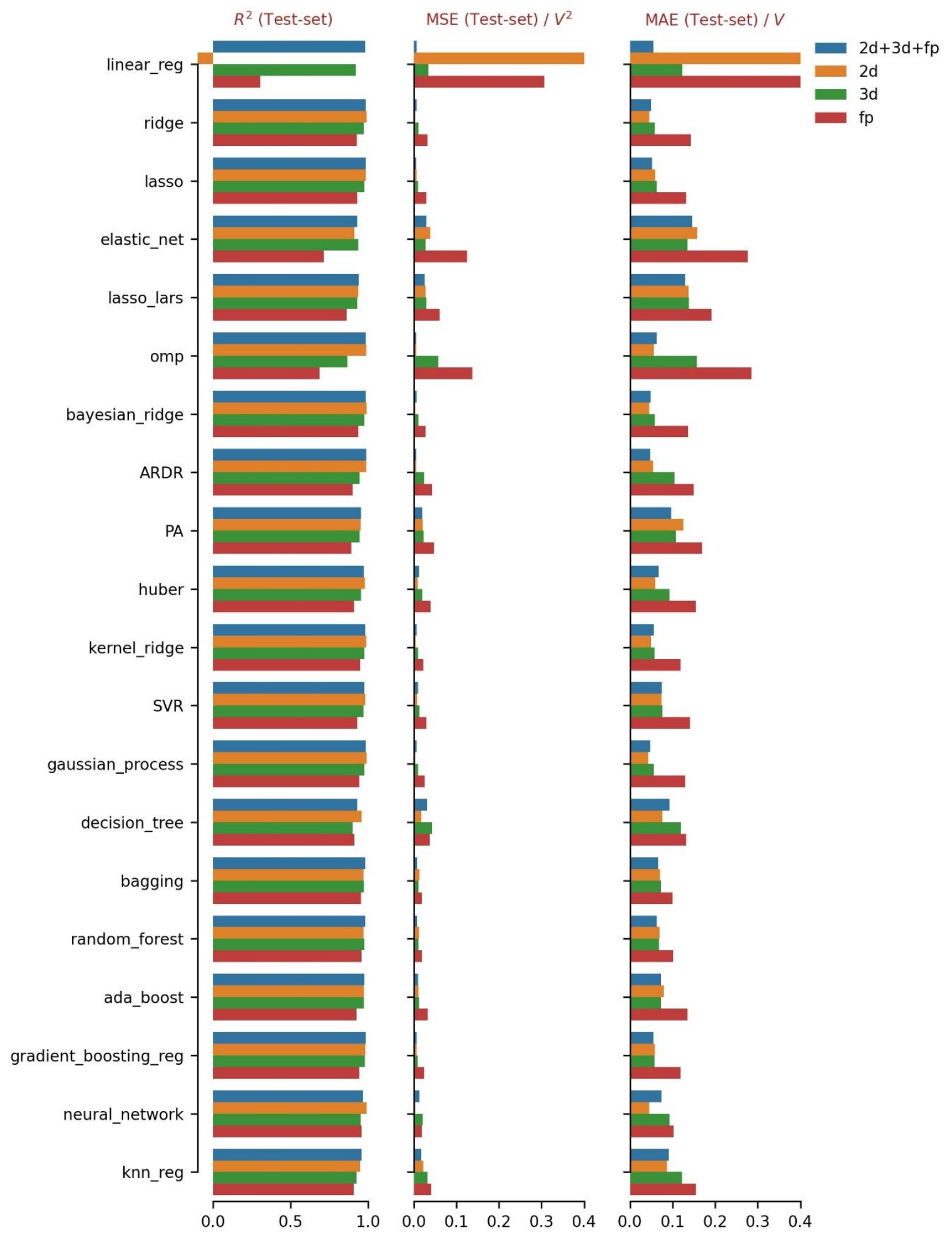

Figure 5. Test-set performance of twenty models. Models were trained on all features from the corresponding feature set. 


\subsection{Feature Importance Analysis}

Here, we performed feature importance analysis for each feature set to identify the most important features. We used Random Forest, AdaBoost, and Gradient Boosting Regression to calculate the feature importance score. Figure 6, Figure 7, Figure 8, and Figure 9 show feature importance histograms for ' $2 d$ ', ' $3 d$ ', ' $f p$ ' and ' $2 d+3 d+f p$ ' feature sets, respectively. Only twenty features with the highest scores are shown in the histograms. The most important features in the ' $2 d$ ' feature set are $S \log P_{-} V S A 4, f r_{-} N H 0, V S A_{-}$Estate3, and VSA_Estate4. SlogP_VSA4 includes the $\log \mathrm{P}^{55}$ and Van der Waals surface area contributions from all atoms in the molecule. $f r \_N H O$ is the number of tertiary amines ${ }^{56}$. VSA_Estate3 and VSA_Estate4 are calculated using EState indices ${ }^{57}$ and Van der Waals surface area contributions of all atoms in the molecule. Many graph-based features like Kappa2, BertzCT, Chil, Chi2n, HallKierAlpha ${ }^{58}$ and some chemically intuitive features like $f r_{-} A r N$ (i.e., number of $\mathrm{N}$ functional groups attached to aromatic ring ${ }^{56}$ ), MinPartialChargge, MaxAbsPartialCharge are also observed in the top twenty features. In the case of ' $3 d$ ' feature set, $R D F_{-} 120, R D F \_90, R D F_{-} 125$ WHIM_90, WHIM_86 are among the top 3D features (RDF, WHIM are $1 \mathrm{D}$ vectors).$^{59}$ The number at the end of the feature name denotes its position in the corresponding feature vector. Some components of MORSE and GETAWAY feature vectors ${ }^{59}$ are also observed in the top twenty features. Only two components of Autocorr3D were observed in one of the histograms (i.e., AdaBoost histogram), suggesting that Autocorr $3 D^{59}$ is a relatively less important $3 \mathrm{D}$ feature. None of the scalar $3 \mathrm{D}$ features were observed in feature importance histograms, suggesting that scalar 3D features are less important than vector 3D features. We had only three types of fingerprints in the ' $f p$ ' feature set (i.e., RDKit, ECFP4, MACCS keys). RDKit Fingerprints are daylight-like fingerprints computed from hashing molecular subgraphs. ${ }^{56}$ ECFP $^{60}$ or Extended Connectivity Circular 
Fingerprints are computed from the bag-of-word representation of the local molecular neighborhood. Four in ECFP4 denotes the radius of local neighborhood. MACCS keys are computed using the SMARTS-based implementation of the 166 public MACCS keys. ${ }^{61}$ Components only from RDKit and ECFP4 were among the top features, whereas only one component from the MACCS keys was observed in one of the histograms (the Gradient Boosting Regression histogram). This indicates that the MACCS key fingerprint does not contain enough molecular information to predict the redox potential. Figure 9 shows the feature importance histograms for the ' $2 d+3 d+f p$ ' feature set. This feature set contains all the features from the ' $2 d$ ', ' $3 d$ ', and ' $f p$ ' feature sets. The most important features in this feature set were also the most important features in their respective set. Top features are mainly from ' $3 d$ ' and ' $2 d$ ' feature sets, and only one component from the ECFP4 feature vector was observed in the lower end of the Gradient Boosting Regression histogram. This again shows that molecular fingerprints are the least informative among all features. The feature importance histogram of the ' $2 d+3 d+f p$ ' feature set contains a few ' $2 d$ ' features and predominantly ' $3 d$ ' features. This can be attributed to how fast importance scores diminish from most important features to the least important features. Feature importance scores in the ' $2 d$ ' feature set (Figure 6) diminish faster than the ' $3 d$ ' feature set (Figure 7). This also indicates that very a few 2D features are required to predict the redox potential compared to $3 \mathrm{D}$ features. 

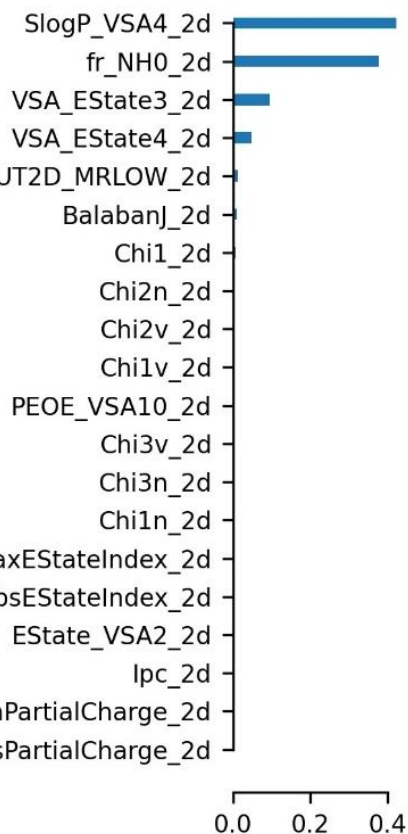

AdaBoost Score

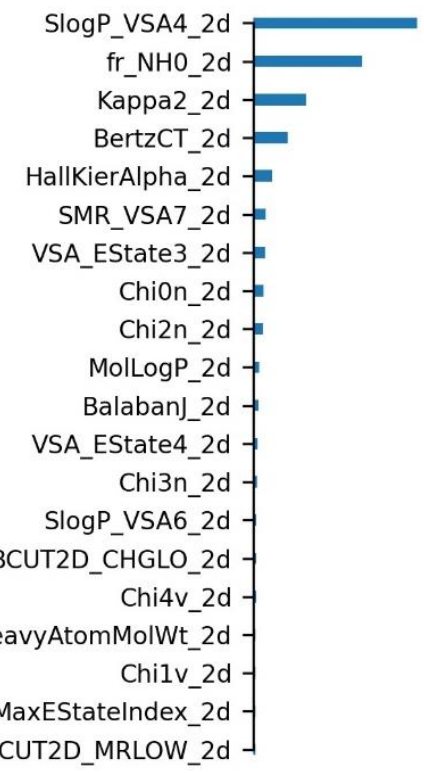

0.00 .10 .20 .3 regression score

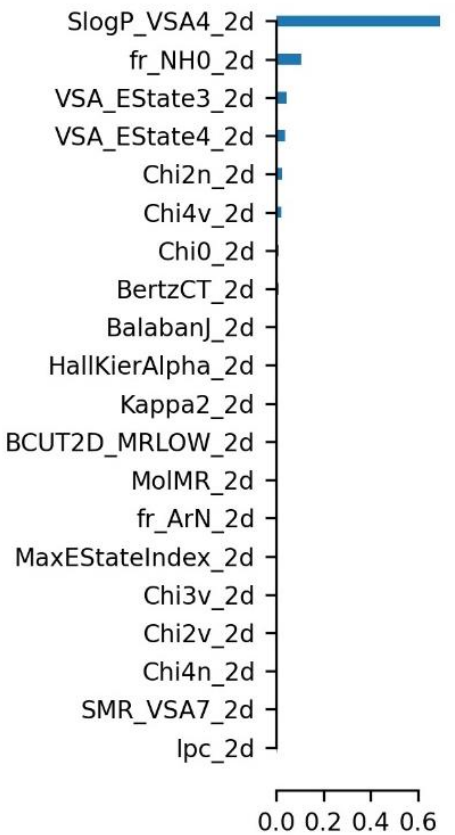

Figure 6. Feature importance histograms of ' $2 d$ ' feature set.
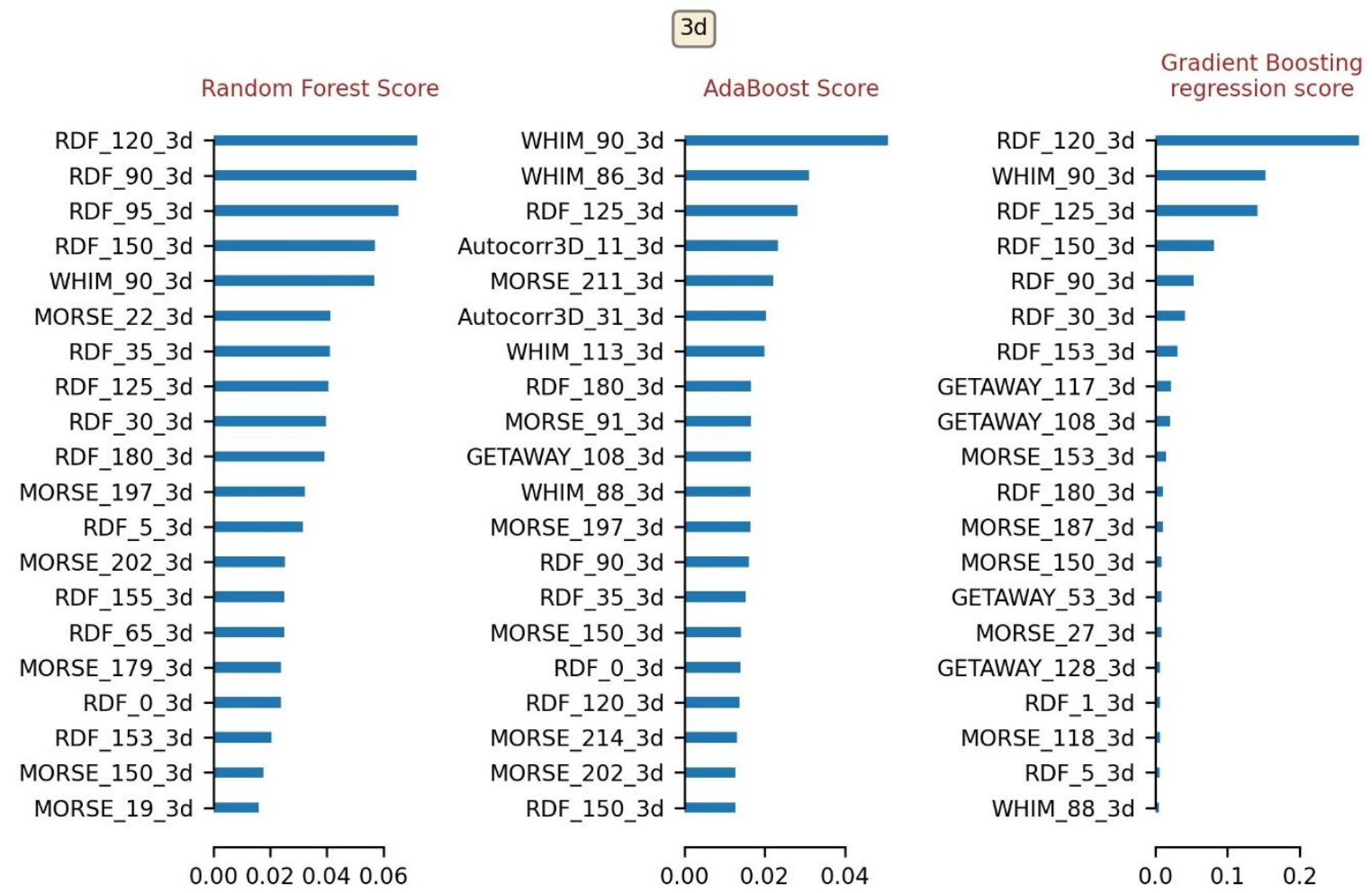

Figure 7. Feature importance histograms of ' $3 d$ ' feature set. 
Random Forest Score

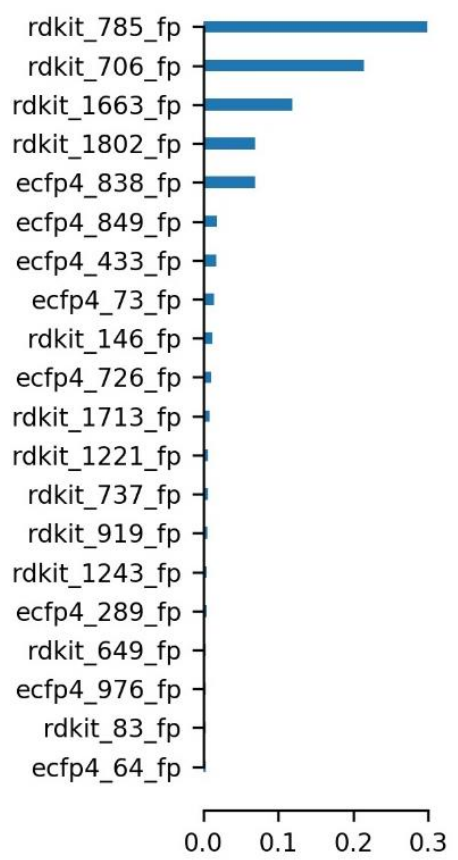

AdaBoost Score

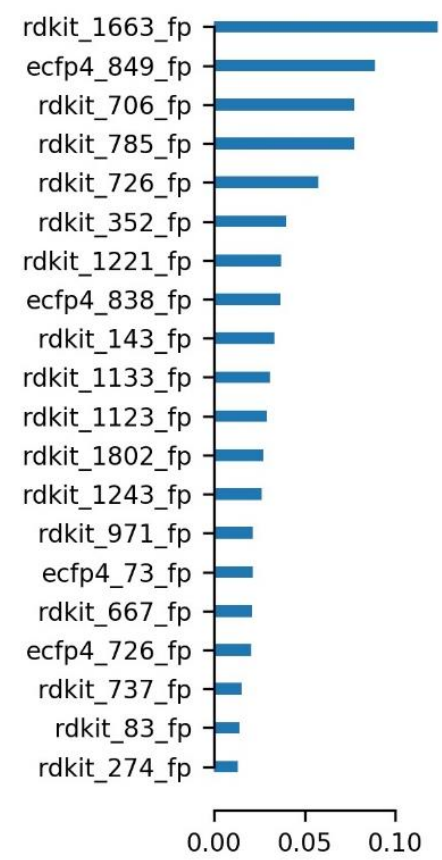

Gradient Boosting regression score

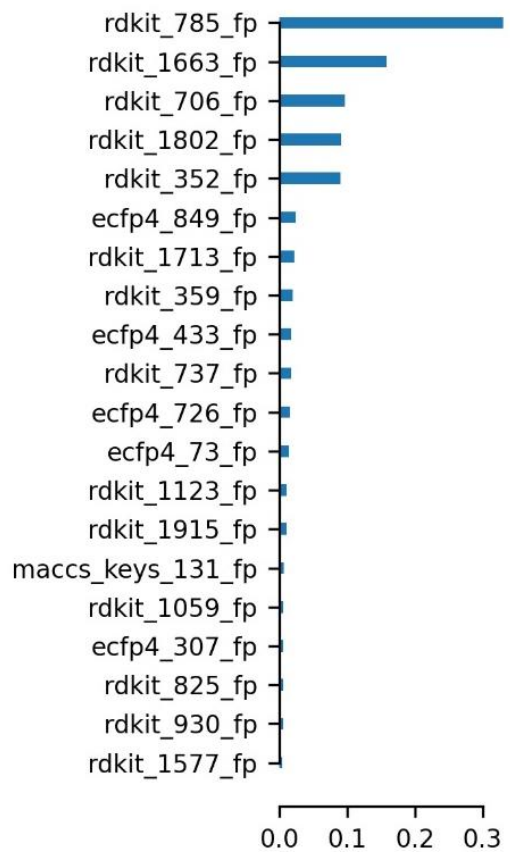

Figure 8. Feature importance histograms of ' $f p$ ' feature set.
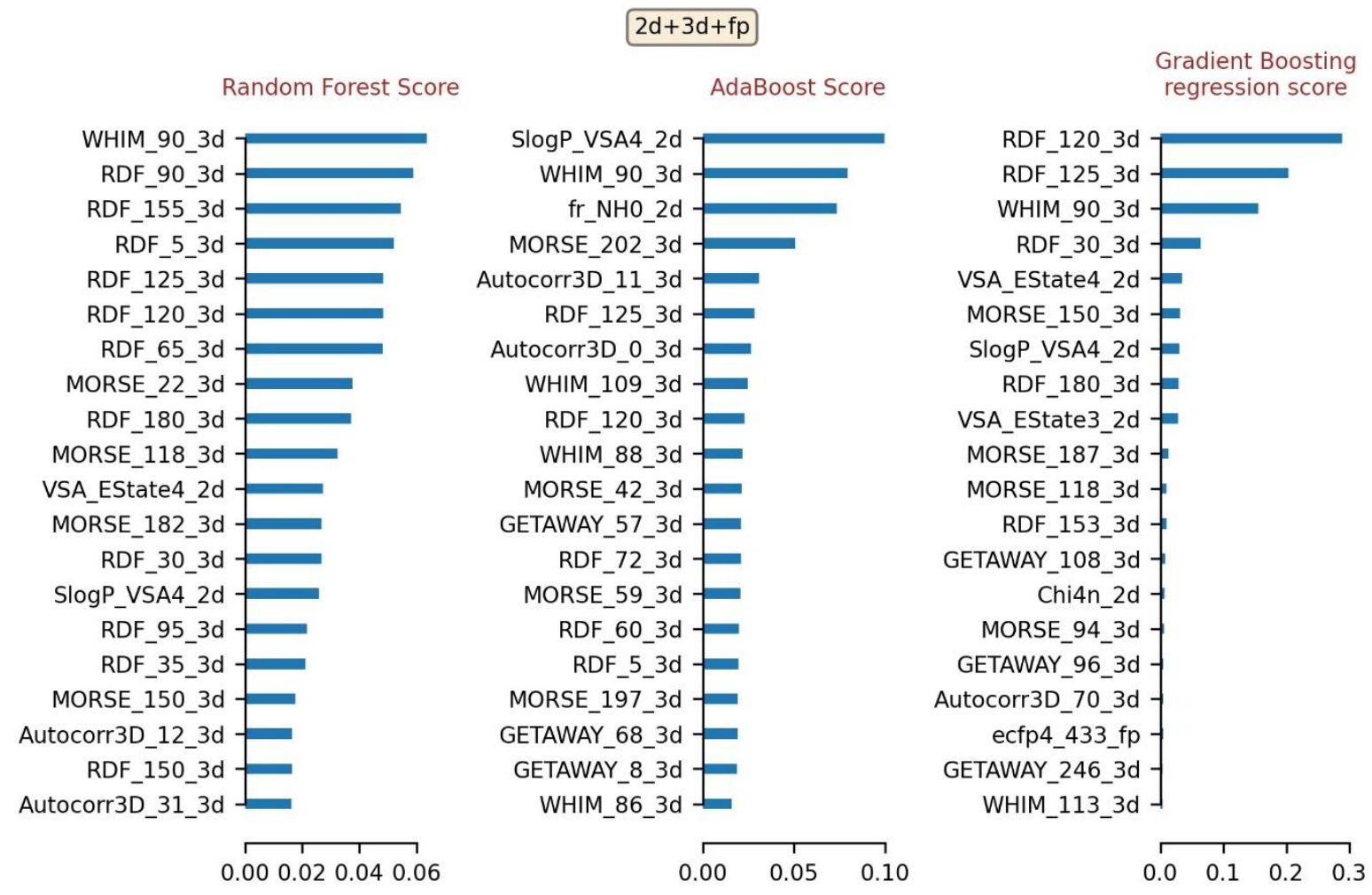

Figure 9. Feature importance histograms of ' $2 d+3 d+f p$ ' feature set. 


\subsection{Effect of Feature Size on Model Performance}
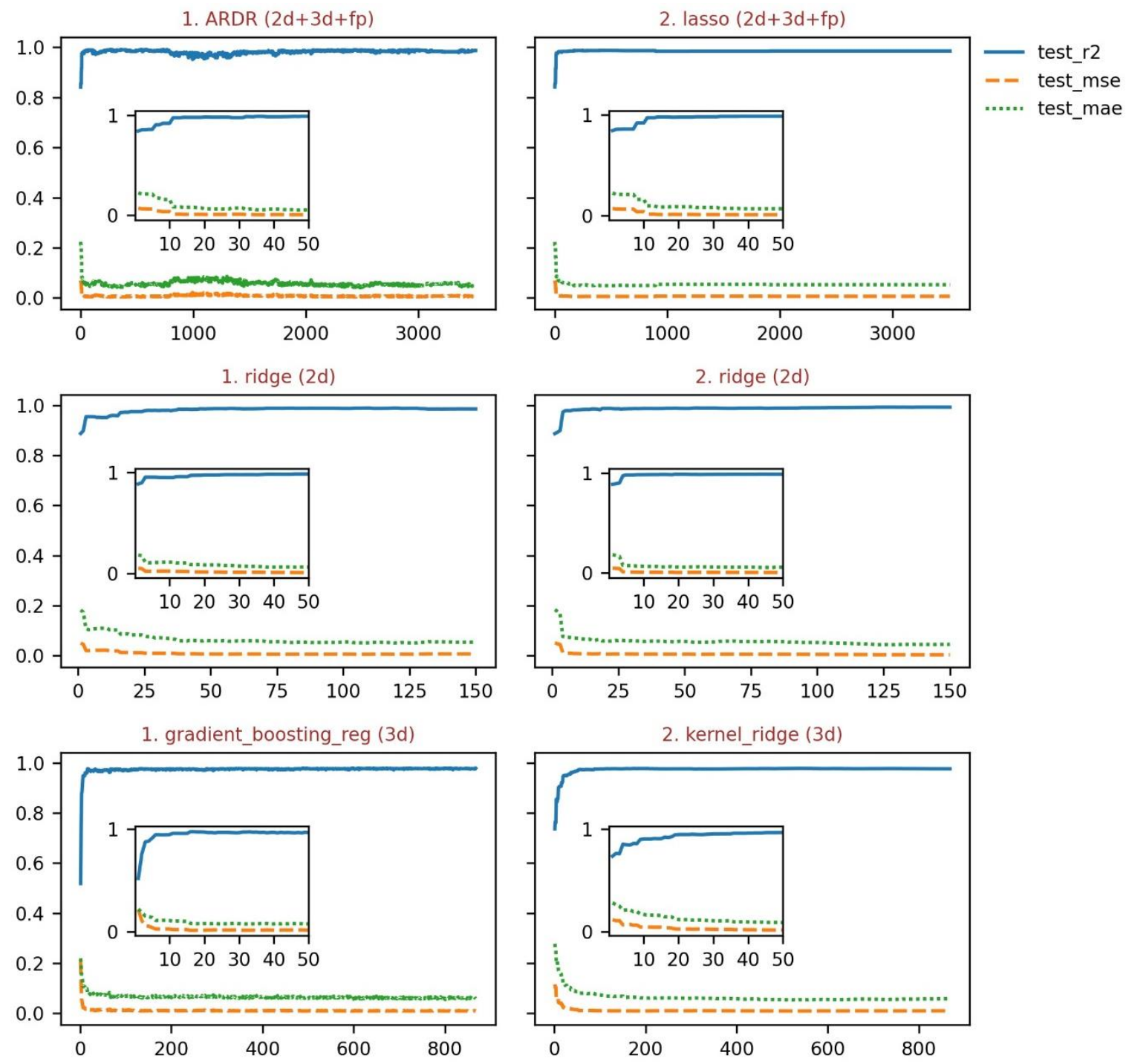

1. random_forest (fp)

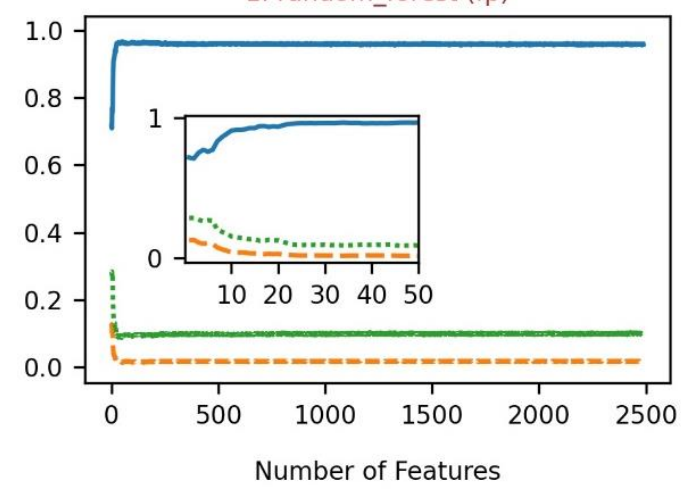

2. neural_network (fp)

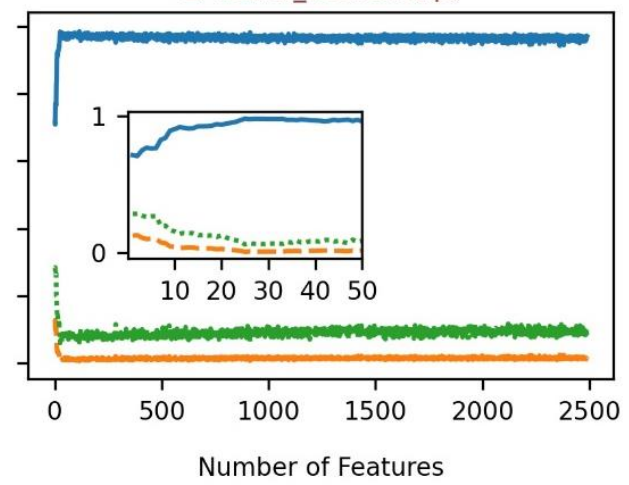

Figure 10. Model performance vs. Number of features 
Previous feature importance studies suggest that not all features may be necessary to achieve high predictive performance. To confirm this hypothesis, two best-performing models in each feature set were selected and re-trained on the subset of features. Features were sorted in descending order based on the Random Forest scores. Models were re-trained starting with a single feature to the complete set of features, and three metrics (i.e., $\mathrm{R}^{2}$, MSE, MAE) on the testset were recorded. The results for each feature set are shown in Figure 10. Inset plots show the same data for the first 50 features. Plots corresponding to the ' $2 d$ ' feature set quickly saturate (after $\sim 5$ features), suggesting that only a small number of $2 \mathrm{D}$ features are required to predict the redox potential accurately. Plots of the ' $3 d$ ' feature set seem to saturate after twenty features, whereas plots corresponding to the ' $f p$ ' feature set saturate slowly and require more than twenty features to achieve similar performance. In the case of the ' $2 d+3 d+f p$ ' feature set, plots seem to saturate around 15-20 features and look approximately similar to a linear combination of the plots from the ' $2 d$ ', ' $3 d$ ', and ' $f p$ ' feature sets. These plots clearly show that not all features are required to attain a high level of prediction accuracy.

\subsection{Assessment of Model Performance on Limited Number of Features}

To gain insight into the quality of predictions when models are trained on the limited number of features, all models were re-trained on the subset of features using the pipeline (see the materials and methods section). Features were chosen from the array of features sorted based on the Random Forest score. The number of features was varied from five to twenty in a step of five. Figure 11 shows the test-set performance of models when trained on a small number of features from the ' $2 d+3 d+f p$ ' feature set. Performance of the full set of features is also shown for reference. We observed that the model performance generally increases with the number of 
features. A few exceptions were also observed. Some models (e.g., PA, huber, neural network, and knn_reg) had better performance on the top twenty features than a full set of features. Moreover, decision_tree performed slightly better on the top fifteen features than a full set of features. Similar trends were observed for other feature sets as well (Figures S2-S4). However, in the case of the ' $f p$ ' feature set, SVR and knn_reg showed better performance on the top twenty features than a full set of features. We also analyzed which feature set was able to achieve the highest accuracy when models were trained on a small number of features. Figure 12 shows the test-set performance of all models when trained on only the top five features from each feature set. ' $2 d$ ' feature set achieved an $\mathrm{R}^{2}$ value as high as 0.9869 with only five features with the 'bagging' model. The ' $2 \mathrm{~d}+3 \mathrm{~d}+\mathrm{fp}$ ' and ' $3 \mathrm{~d}$ ' feature set performance was similar but sub-par to the ' $2 d$ ' feature set. The similarity in performance of ' $2 d+3 d+f p$ ' and ' $3 d$ ' feature sets could be attributed to the similarity in their top five features (see Figure 7 and Figure 9). The ' $f p$ ' feature set performed poorly with only five features. These results were consistent across all models. Similar results were also obtained for the models trained on the top ten, fifteen, and twenty features are shown in Figures S5-S7. Furthermore, a significant decrease in the training and inference time was observed while using the limited number of features for the training. These results suggest that training and inference time could be reduced by using a small number of features while maintaining a good accuracy level. 


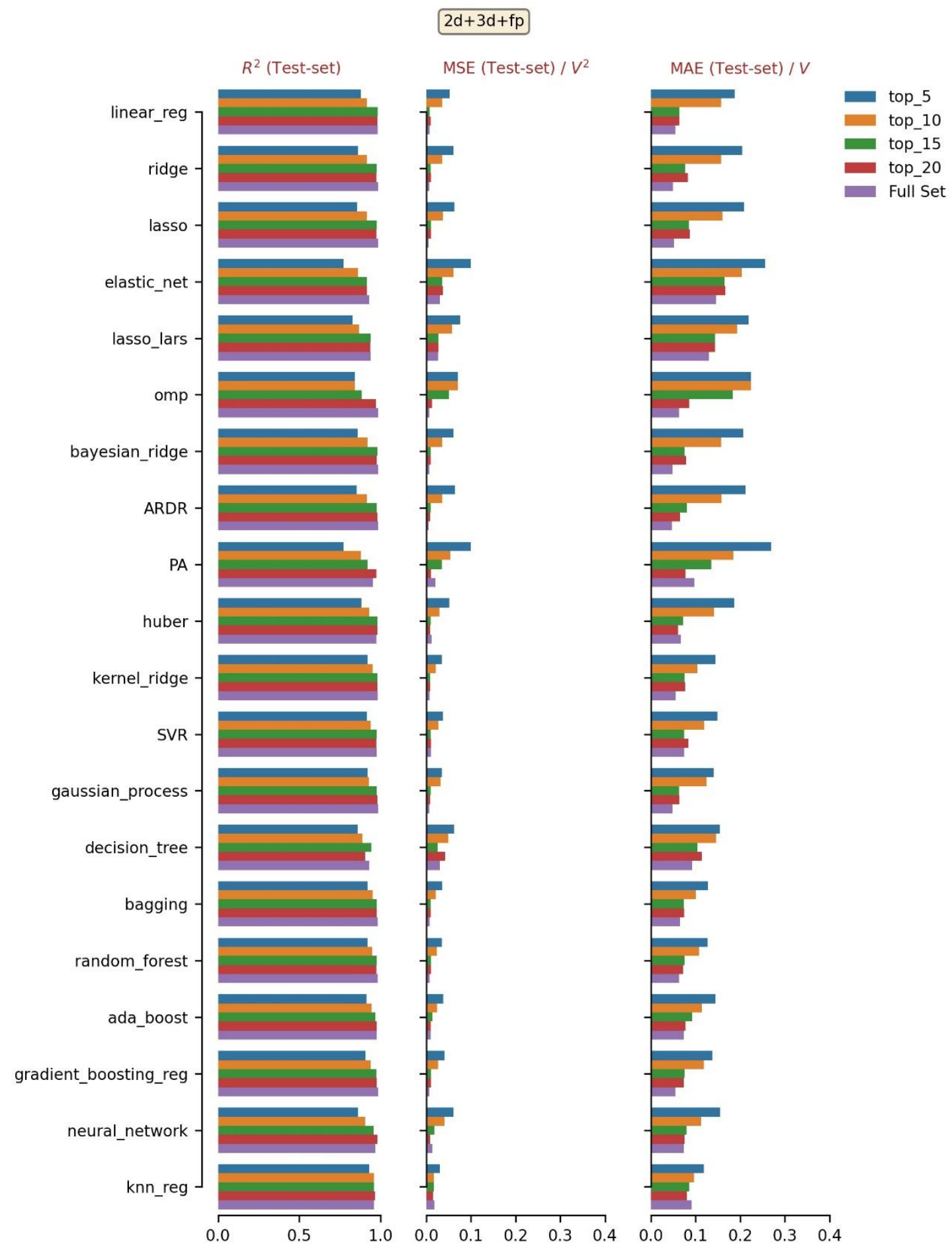

Figure 11. Test-set performance of twenty models trained on top-5, 10, 15, and 20 features from ' $2 d+3 d+f p$ ' feature set. Top most important features were selected based on the Random Forest score. Full feature set performance is shown for the reference. 


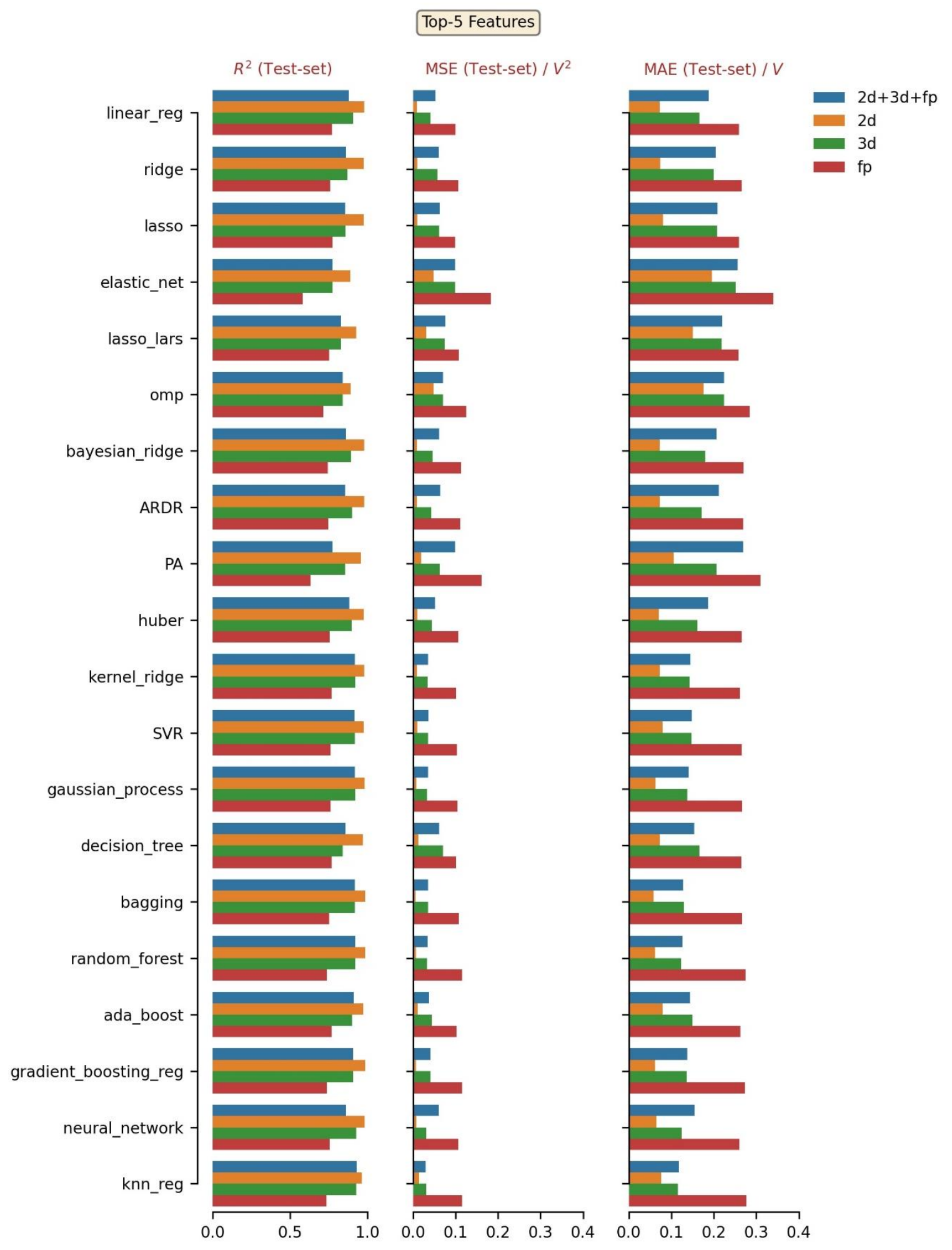

Figure 12. Test-set performance of twenty models trained on five most important features from the corresponding feature set. Features were selected based on the Random Forest score. 


\subsection{Identification of the Promising Redox-Active Candidates}

Compounds having a significant difference in their redox potential are considered to be promising redox-active species. In this study, the trained machine learning models were able to predict previously reported promising redox-active candidates. In particular, cathode redoxactive compounds such as tetra-amino-phenazine (TAPZ), hexa-amino-phenazine (HAPZ), and octa-amino-phenazine (OAPZ) were predicted with high accuracy (using the best-performing model). The absolute difference between predicted and actual redox potential of TAPZ, HAPZ, and OAPZ derivatives was less than $0.07 \mathrm{~V}(<3 \%)$. Furthermore, our best-performing model was able to predict the redox potential of octa-nitro-phenazine (ONPZ) with an error less than $0.004 \mathrm{~V}(<2.4 \%)$, even though the dataset used in this study contains only a few compounds with positive redox potential. ONPZ is considered a promising anode redox-active candidate for RBFs containing phenazine derivatives. ${ }^{18}$ Additionally, most models were able to predict the correct relative order of compounds with respect to the redox potential, clustering potential cathode redox-active candidates in the negative region, and potential anode redox-active candidates in the region close to zero. It can be seen that the promising cathode redox-active species contain electron-donating groups (i.e., $\mathrm{NH}_{2}$ ), whereas promising anode redox-active species contain electron-withdrawing groups (i.e., $\mathrm{CN}, \mathrm{NO}_{2}$ ). These results are in good agreement with the previous report. ${ }^{18}$

\subsection{Analysis of the Predictive Performance on Individual Function Groups}

Here, we evaluate the prediction accuracy of the best-performing models in each feature set with respect to different functional groups attached to the phenazine ring. Twenty different functional groups were present in the phenazine derivatives used in this study. Train-set and 
Test-set Predictions were obtained from the best performing model in each feature set (see Table 5). Figure 13 shows the Mean Absolute Percentage Error (MAPE) for each functional group (FG) present in the test-set. MAPE in Figure 13a is averaged over FGs and four feature sets.

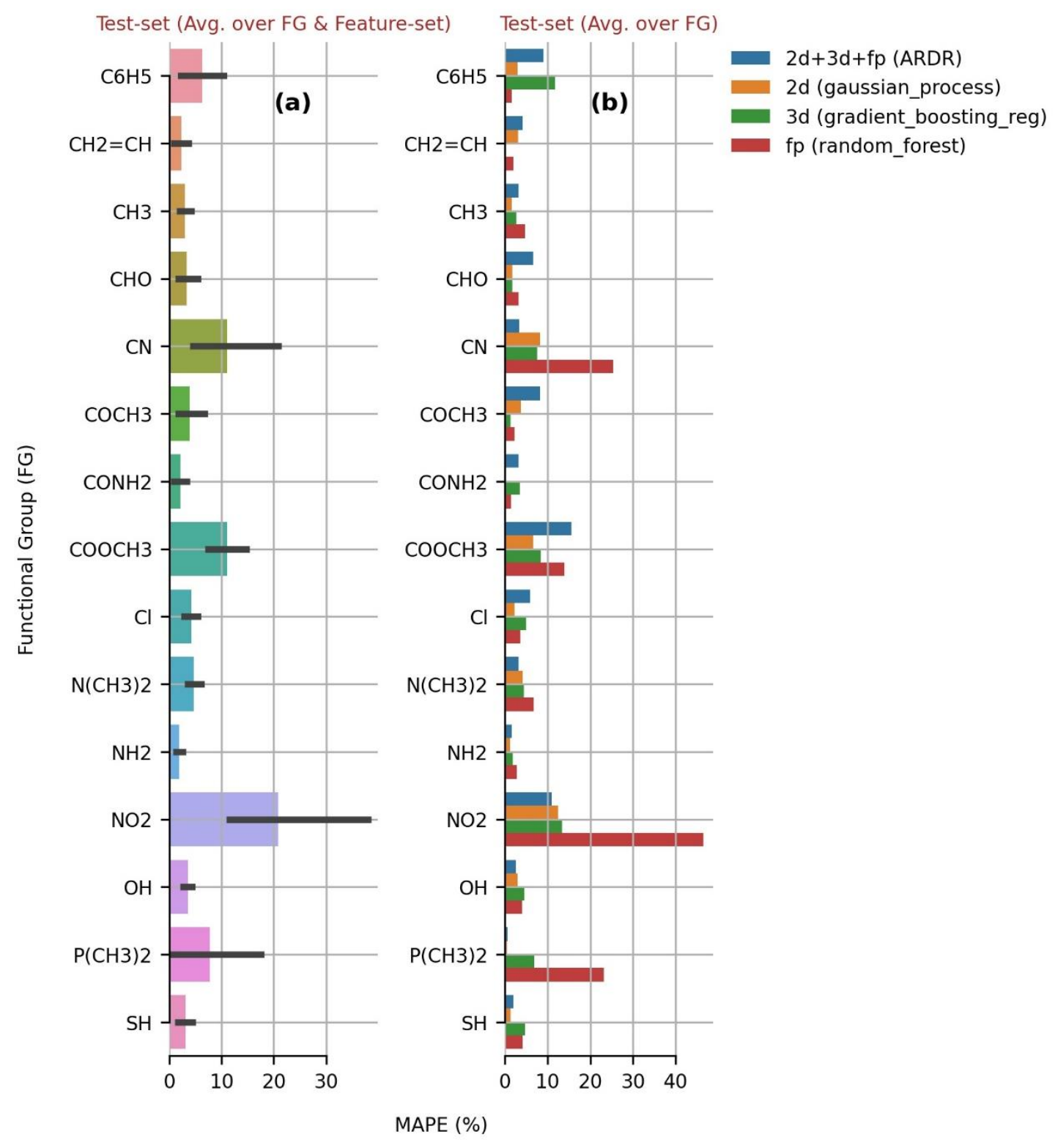

Figure 13. Functional group (FG) vs. Mean Absolute Percentage Error (MAPE) of testset. (a) MAPE is averaged over FG and feature sets. (b) MAPE is averaged over FG. Testset predictions were obtained from the best-performing model in the corresponding 
On the other hand, MAPE, shown in Figure $13 \mathrm{~b}$ is averaged over only FGs. MAPE of the functional groups present in the train-set is shown in Figure S8. MAPE for the majority of the functional groups was well below 10.0\%. Even though some functional groups appeared only once in the training set (see Figure 14), most of the models were able to predict the redox potential with minimal error. The functional group $\mathrm{COCH}_{3}$ was present only in the test-set, which means that models never saw this functional group during the training. Still, the error in its prediction was less than $5.0 \%$. This shows that models successfully learned the hidden patterns between features and redox potential from the training set, resulting in low generalization errors.

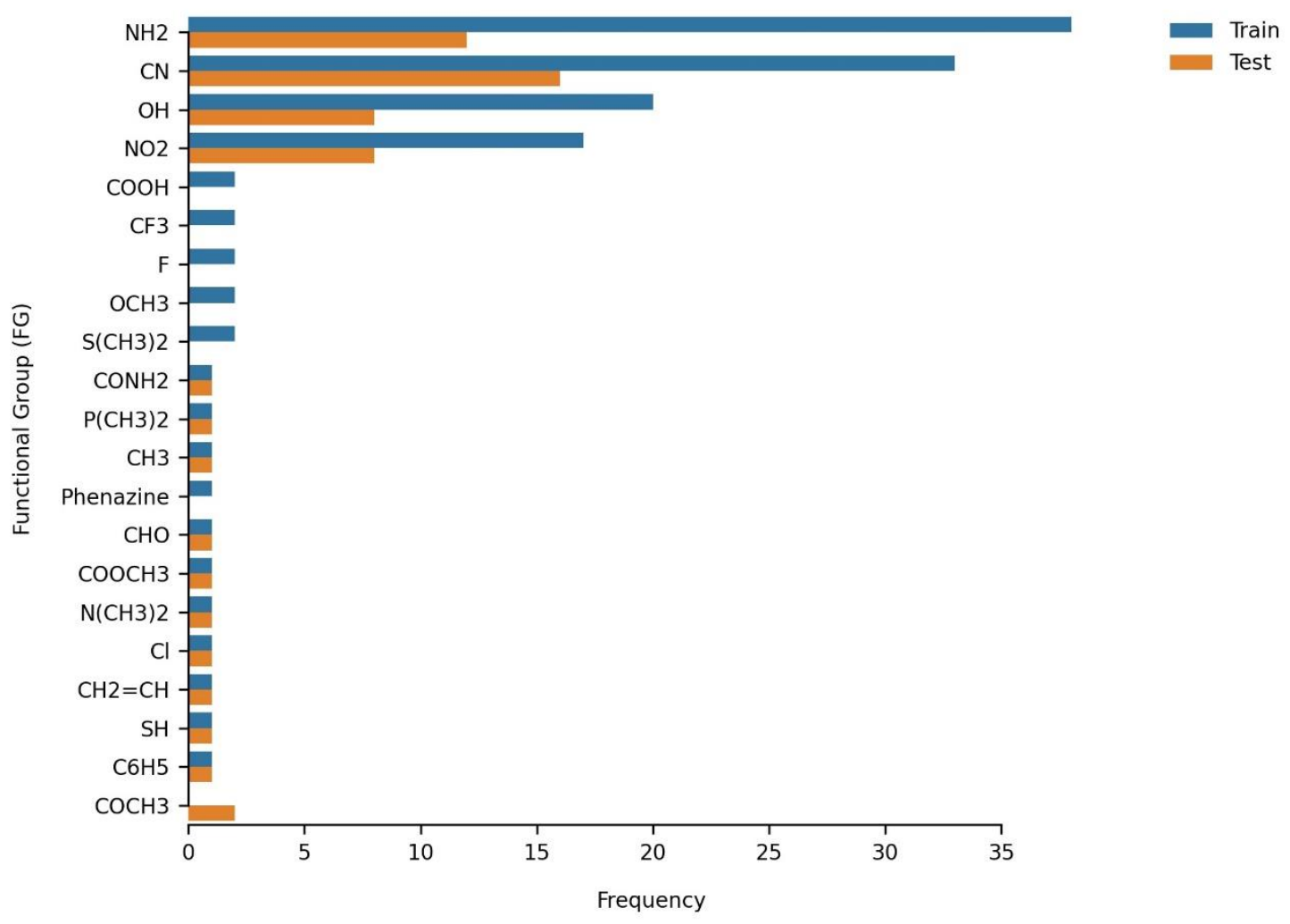

Figure 14. Distribution of functional groups in train and test sets. 


\subsection{Error Analysis}

Even though models had low generalization errors, some functional groups exhibited relatively high MAPE, e.g., $\mathrm{CN}, \mathrm{NO}_{2}$, and $\mathrm{COOCH}_{3}$. High errors of $\mathrm{CN}$ and $\mathrm{NO}_{2}$ are attributed to a small number of compounds with the redox potential close to zero. Unfortunately, the data used in this study contains a very small number of compounds with redox potential close to zero. The whole dataset contains only 18 compounds $(\sim 9.7 \%$ of the total data) having redox potential greater than $-0.5 \mathrm{~V}$ (Figure 15a). Therefore, models had less information to learn from in the region near zero redox potential. This is the reason why test-set predictions near-zero redox potential had relatively high errors (Figure 16). On the other hand, having access to a large enough dataset in the region below $-0.5 \mathrm{~V}$, models were able to learn the hidden patterns. This resulted in low prediction errors for the compounds with redox potential below $-0.5 \mathrm{~V}$, even for compounds with less than one sample in the train-set. Functional groups $\mathrm{CN}$ and $\mathrm{NO}_{2}$ contain some compounds with redox potential greater than $-0.5 \mathrm{~V}$ (see Figure 13b), responsible for the high prediction errors observed in Figure 13. We also observed a slight increase in the errors around $-1.5 \mathrm{~V}$ in Figure 16, which could be attributed to the relatively low number of data points in the region near $-1.5 \mathrm{~V}$. This why $\mathrm{COOCH}_{3}$ (avg. redox potential $-1.57 \mathrm{~V}$ ) also showed a slightly high prediction error. The red curve in Figure 16 shows the normalized distribution over redox potential (i.e., density) for the whole dataset. 


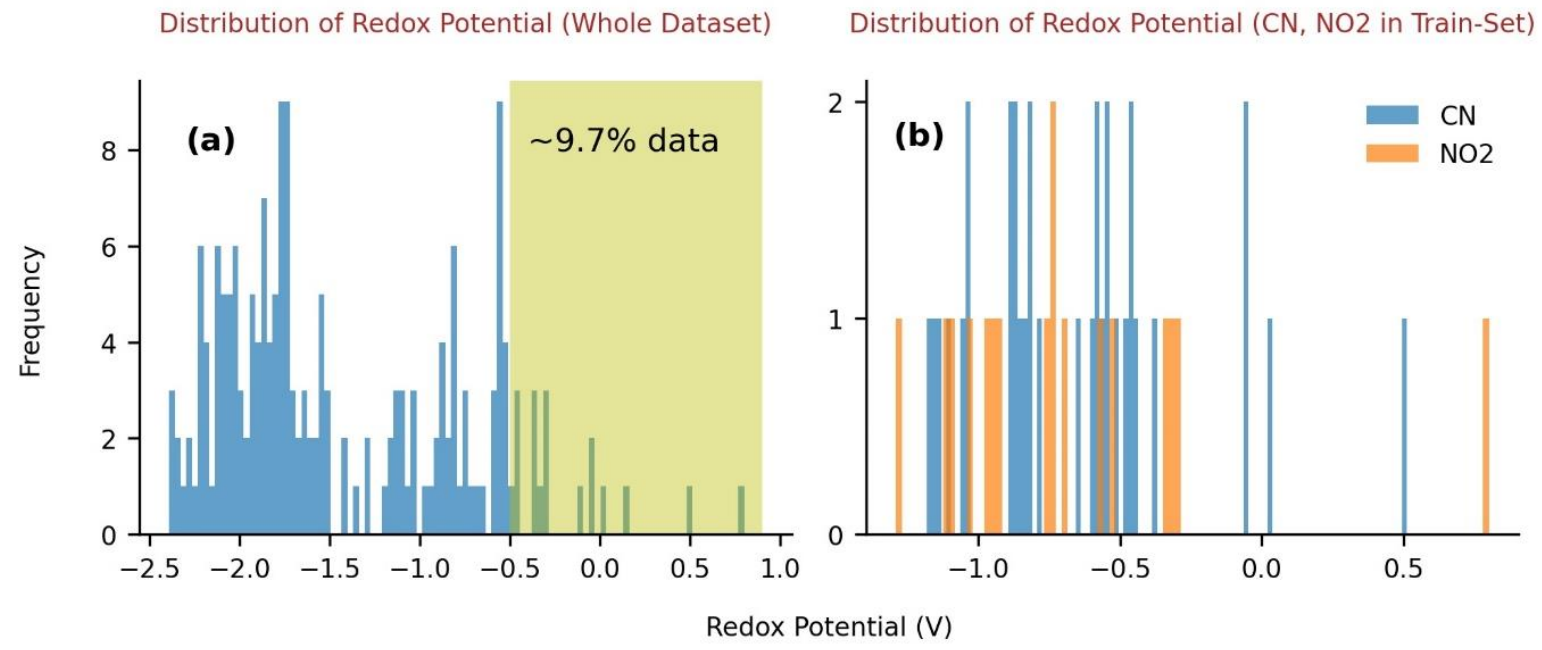

Figure 15. Distribution of redox potential (a) of whole dataset. (b) of $\mathrm{CN}, \mathrm{NO}_{2}$ functional groups in train-set

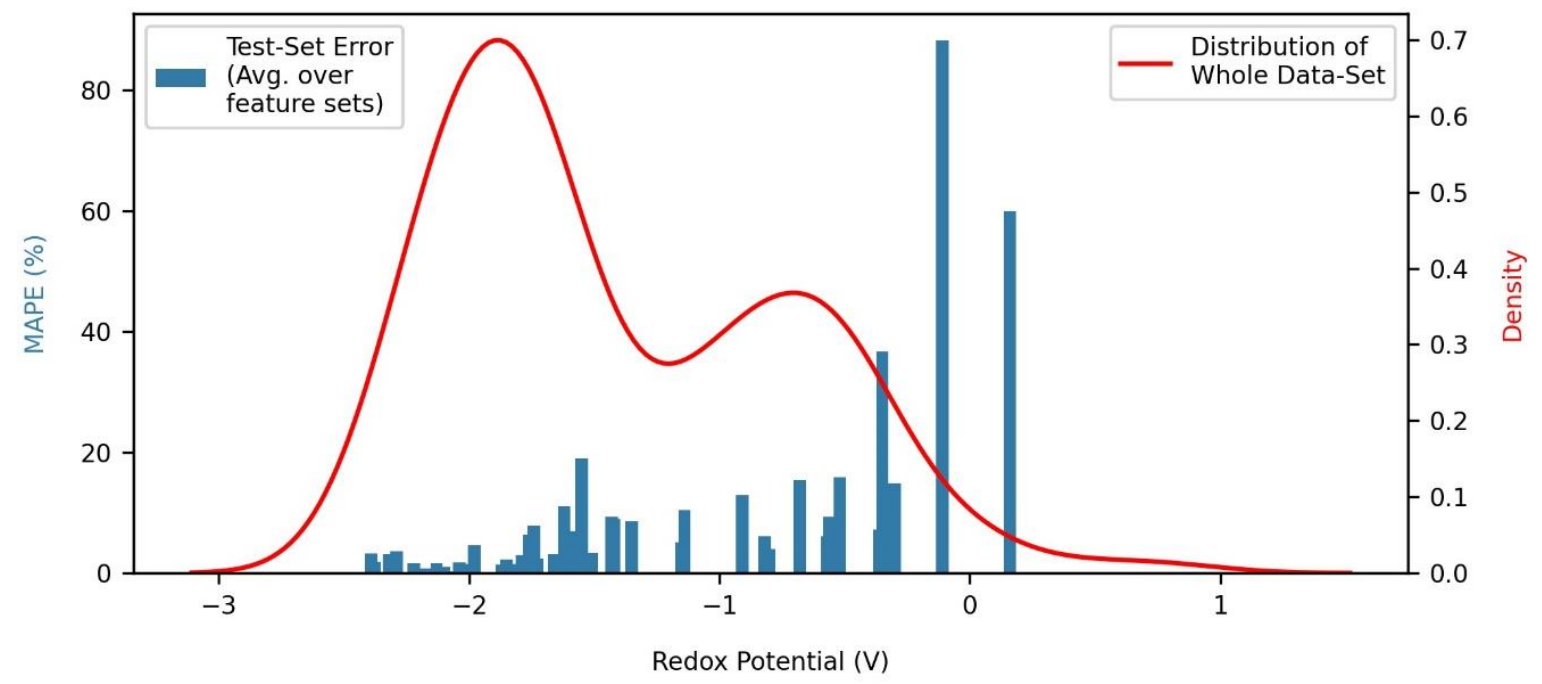

Figure 16. MAPE vs. redox potential. Final MAPE on the y-axis was calculated by averaging the MAPE obtained from the best-performing model in each feature set. 


\section{CONCLUSIONS}

In this study, we have investigated twenty linear and non-linear machine-learning models to predict the redox potential of phenazine derivatives in DME. Both linear and non-linear models trained on a small dataset were able to achieve excellent prediction accuracy on the test-set (i.e., $\mathrm{R}^{2}>0.98, \mathrm{MSE}<0.008 \mathrm{~V}^{2}$, and MAE $<0.07 \mathrm{~V}$ ). Features used in this study were intentionally chosen to be easily computable from open-source libraries that do not require DFT calculations or experimental measurements, making our approach readily adaptable for similar studies. Model performance was assessed on four feature sets containing different features (i.e., 2D, 3D, and Molecular Fingerprints) using a convenient pipeline developed in this work. This pipeline combines different training and evaluation components in a single sub-subroutine, making the whole process easy, consistent, and automatic for all models in different scenarios. Gaussian Processes Regression trained on 2D features achieved the highest prediction accuracy. The analysis of model performance on four feature sets revealed an interesting order with respect to prediction accuracy: $2 d>2 d+3 d+f p>3 d>f p$. Average performance analysis also showed that 2D features are better at generalizing to unseen data than 3D and molecular fingerprint features. Therefore, we conclude that 2D features capture important molecular properties necessary for predicting the redox potential of phenazine derivatives in DME solvent. It was observed that linear models out-perform non-linear models on ' $2 d+3 d+f p$ ' and ' $2 d$ ' feature sets, whereas nonlinear models perform better than linear models on ' $2 d$ ', ' $3 d$ ' and ' $f p$ ' feature sets. Therefore, linear models should be preferred when the feature set contains different types of features, and non-linear models should be preferred when the feature set contains either 3D or molecular fingerprint features. Due to the simple structure, linear models have fast training and inference time but suffer from low accuracy. Results obtained here show that lower training and inference 
times are possible to achieve with the linear models that out-perform non-linear models when dataset contains different types of features (i.e., 2D, 3D, and Molecular Fingerprints). Feature importance analysis showed that features related to Van der Waals surface areas, e.g., SlogP_VSA4, fr_NHO,VSA_Estate3, and VSA_Estate4 were the most important 2D features. RDF_120, RDF_90, RDF_125, WHIM_90, and WHIM_86 were the most important 3D features. RDKit, ECFP4 were the most important molecular fingerprint features. Some features based on molecular structure and charges, e.g., fr_ArN, MinPartialChargge, and MaxAbsPartialCharge, were also observed during feature importance analysis. Feature importance analysis also suggested that very few $2 \mathrm{D}$ features are required to predict the redox potential compared to $3 \mathrm{D}$ and molecular fingerprint features. This observation was confirmed by re-training models with the subset of features starting from a single feature to a full set of features. Model performance was generally observed to increase with the number of features, but some exceptions were also observed for which the small number of features performed better than a full set of features. A bagging meta-estimator trained on only the top five 2D most important features was able to achieve $\mathrm{R}^{2}$ value as high as 0.9869 . Significant reduction in the training and inference time was observed while maintaining a good level of accuracy. Thus, results obtained in this study would also help in reducing the training and inference time for similar future studies on large datasets. MAPE for most functional groups was well below $10.0 \%$, even for the functional groups with one or zero compounds in the training set. This shows that models were able to successfully learn hidden patterns and generalize quite well to the unseen data. High test errors for three functional groups $\left(\mathrm{CN}, \mathrm{NO}_{2}\right.$, and $\left.\mathrm{COOCH}_{3}\right)$ were observed due to the small number of data points in the region around their average redox potential. With the machine-learning models developed in this study, it will be possible to screen millions of new phenazine derivatives in a 
reasonable amount of time compared to experimental or DFT methods. Thus, new compounds from large chemical space can be quickly identified as potential redox-active species in RFBs. Furthermore, these models will reduce the number of compounds that need to be analyzed using DFT calculations in hybrid ML-DFT approaches. Thus, we have shown that machine-learningbased approaches can accelerate the discovery of novel materials for energy storage applications.

\section{FUTURE DIRECTIONS}

Data used in this study contained only twenty functional groups. Furthermore, redox potentials

were obtained in only DME solvent. This restricts the applicability of models to only twenty functional groups and one solvent. Future studies could expand the dataset to include more functional groups and redox potentials determined in various solvents. The primary source of error in this study was a lack of data near zero and in the positive redox potential region. Thus, a large dataset containing compounds with approximately uniform distribution over positive and negative redox potential would significantly reduce the generalization errors. This study uses only phenazine derivatives, limiting the applicability of models to only phenazine derivatives. Future studies could combine various compounds to develop a universal model to predict the redox potential of a variety of compounds. 


\section{ASSOCIATED CONTENT}

\section{Supporting Information}

The Supporting Information is available free of charge.

Tables showing the list of all features, ten best-performing models trained on all features from ' $2 d+3 d+f p$ ', ' $2 d$ ', ' $3 d$ ', and ' $f p$ ' feature sets, best-performing feature set for each of twenty models. Figures showing train-set performance of twenty models, test-set performance of twenty models trained on top-5, 10, 15, and 20 features from ' $2 d+3 d+f p$ ', ' $2 d$ ', ' $3 d$ ', and ' $f p$ ' feature set, functional group (FG) vs. Mean Absolute Percentage Error (MAPE) of train-set. (PDF)

\section{AUTHOR INFORMATION}

\section{Corresponding Authors}

Siddharth Ghule - Physical and Materials Chemistry Division, CSIR-National Chemical Laboratory (CSIR-NCL), Dr. Homi Bhabha Road, Pashan, Pune 411008, India; Academy of Scientific and Innovative Research (AcSIR), Ghaziabad 201002, India; ORCID iD: https://orcid.org/0000-0003-0864-0777; Email: ss.ghule@ncl.res.in; Phone: +91-20-25903095

Kumar Vanka - Physical and Materials Chemistry Division, CSIR-National Chemical Laboratory (CSIR-NCL), Dr. Homi Bhabha Road, Pashan, Pune 411008, India; Academy of Scientific and Innovative Research (AcSIR), Ghaziabad 201002, India; ORCID iD: http://orcid.org/0000-00017301-7573; Email: k.vanka@,ncl.res.in; Phone: +91-20-25903095 


\section{Authors}

Sayan Bagchi - Physical and Materials Chemistry Division, CSIR-National Chemical Laboratory (CSIR-NCL), Pune, 411008, India; Academy of Scientific and Innovative Research (AcSIR), Ghaziabad 201002, India

\section{Author Contributions}

The manuscript was written through contributions of all authors. All authors have given approval to the final version of the manuscript.

\section{Notes}

The authors declare no competing financial interest.

\section{DATA AND CODE AVAILABILITY}

The training and test datasets along with the jupyter notebook used for model training is available at https://github.com/siddharth-ncl-work/ML-Redox-Potential.git

\section{ACKNOWLEDGMENTS}

K.V. is grateful to the Department of Science and Technology (DST) (EMR/2014/000013) for providing financial assistance. S.B. acknowledges SERB India (EMR/2016/000576). S.G. thanks Council of Scientific and Industrial Research (CSIR) for providing Research Fellowship. The support and the resources provided by 'PARAM Brahma Facility' under the National Supercomputing Mission, Government of India at the Indian Institute of Science Education and Research (IISER) Pune are gratefully acknowledged. 


\section{REFERENCES}

(1) Shafiee, S.; Topal, E. When Will Fossil Fuel Reserves Be Diminished? Energy Policy 2009, 37 (1), 181-189. https://doi.org/10.1016/j.enpol.2008.08.016.

(2) Dehghani-Sanij, A. R.; Tharumalingam, E.; Dusseault, M. B.; Fraser, R. Study of Energy Storage Systems and Environmental Challenges of Batteries. Renew. Sustain. Energy Rev. 2019, 104 (January), 192-208. https://doi.org/10.1016/j.rser.2019.01.023.

(3) Höök, M.; Tang, X. Depletion of Fossil Fuels and Anthropogenic Climate Change-A Review. Energy Policy 2013, 52, 797-809. https://doi.org/10.1016/j.enpol.2012.10.046.

(4) Gür, T. M. Review of Electrical Energy Storage Technologies, Materials and Systems: Challenges and Prospects for Large-Scale Grid Storage. Energy Environ. Sci. 2018, 11 (10), 2696-2767. https://doi.org/10.1039/c8ee01419a.

(5) Chu, W. S.; Chun, D. M.; Ahn, S. H. Research Advancement of Green Technologies. Int. J. Precis. Eng. Manuf. 2014, 15 (6), 973-977. https://doi.org/10.1007/s12541-014-0424-8.

(6) Balat, H. Green Power for a Sustainable Future. Energy Explor. Exploit. 2007, 25 (1), 125. https://doi.org/10.1260/014459807781036403.

(7) Demirbas, A. Electrical Power Production Facilities from Green Energy Sources. Energy Sources, Part B Econ. Plan. Policy 2006, $1 \quad$ (3), 291-301. https://doi.org/10.1080/15567240500400648.

(8) Dunn, B.; Kamath, H.; Tarascon, J. M. Electrical Energy Storage for the Grid: A Battery of Choices. Science (80-. ). 2011, 334 (6058), 928-935. https://doi.org/10.1126/science.1212741. 
(9) Chung, E. What caused the deadly power outages in Texas and how Canada's grid compares https:/www.cbc.ca/news/technology/power-outages-texas-canada-1.5920833 (accessed Mar 30, 2021).

(10) Larcher, D.; Tarascon, J. M. Towards Greener and More Sustainable Batteries for Electrical Energy Storage. Nat. Chem. 2015, 7 (1), 19-29. https://doi.org/10.1038/nchem.2085.

(11) Koohi-Fayegh, S.; Rosen, M. A. A Review of Energy Storage Types, Applications and Recent Developments. Journal of Energy Storage. Elsevier Ltd February 1, 2020, p 101047. https://doi.org/10.1016/j.est.2019.101047.

(12) Deng, D. Li-ion Batteries: Basics, Progress, and Challenges. Energy Sci. Eng. 2015, 3 (5), 385-418. https://doi.org/10.1002/ese3.95.

(13) Skyllas-Kazacos, M.; Chakrabarti, M. H.; Hajimolana, S. A.; Mjalli, F. S.; Saleem, M. Progress in Flow Battery Research and Development. J. Electrochem. Soc. 2011, 158 (8), R55. https://doi.org/10.1149/1.3599565.

(14) Leung, P.; Li, X.; Ponce De León, C.; Berlouis, L.; Low, C. T. J.; Walsh, F. C. Progress in Redox Flow Batteries, Remaining Challenges and Their Applications in Energy Storage. RSC Adv. 2012, 2 (27), 10125-10156. https://doi.org/10.1039/c2ra21342g.

(15) Sánchez-Díez, E.; Ventosa, E.; Guarnieri, M.; Trovò, A.; Flox, C.; Marcilla, R.; Soavi, F.; Mazur, P.; Aranzabe, E.; Ferret, R. Redox Flow Batteries: Status and Perspective towards Sustainable Stationary Energy Storage. J. Power Sources 2021, 481, 228804. https://doi.org/10.1016/j.jpowsour.2020.228804. 
(16) Alotto, P.; Guarnieri, M.; Moro, F. Redox Flow Batteries for the Storage of Renewable Energy: A Review. Renew. I\& Sustain. ENERGY Rev. 2014, 29, 325-335. https://doi.org/10.1016/j.rser.2013.08.001.

(17) Qi, Z.; Koenig, G. M. Review Article: Flow Battery Systems with Solid Electroactive Materials. J. Vac. Sci. Technol. B, Nanotechnol. Microelectron. Mater. Process. Meas. Phenom. 2017, 35 (4), 040801. https://doi.org/10.1116/1.4983210.

(18) De La Cruz, C.; Molina, A.; Patil, N.; Ventosa, E.; Marcilla, R.; Mavrandonakis, A. New Insights into Phenazine-Based Organic Redox Flow Batteries by Using High-Throughput DFT Modelling. Sustain. Energy Fuels 2020, 4 (11), 5513-5521. https://doi.org/10.1039/d0se00687d.

(19) Gentil, S.; Reynard, D.; Girault, H. H. Aqueous Organic and Redox-Mediated Redox Flow Batteries: A Review. Current Opinion in Electrochemistry. Elsevier B.V. June 1, 2020, pp 7-13. https://doi.org/10.1016/j.coelec.2019.12.006.

(20) Leung, P.; Shah, A. A.; Sanz, L.; Flox, C.; Morante, J. R.; Xu, Q.; Mohamed, M. R.; Ponce de León, C; Walsh, F. C. Recent Developments in Organic Redox Flow Batteries: A Critical Review. Journal of Power Sources. Elsevier B.V. August 31, 2017, pp 243-283. https://doi.org/10.1016/j.jpowsour.2017.05.057.

(21) Cao, J.; Tian, J.; Xu, J.; Wang, Y. Organic Flow Batteries: Recent Progress and Perspectives. Energy and Fuels 2020, $34 \quad$ (11), 13384-13411. https://doi.org/10.1021/acs.energyfuels.0c02855. 
(22) Li, M.; Rhodes, Z.; Cabrera-Pardo, J. R.; Minteer, S. D. Recent Advancements in Rational Design of Non-Aqueous Organic Redox Flow Batteries. Sustain. Energy Fuels 2020, 4 (9), 4370-4389. https://doi.org/10.1039/d0se00800a.

(23) Elena I. Romadina, Denis S. Komarov, K. J. S.; A.Troshin, P. New Phenazine Based Anolyte Material for High Voltage Organic Redox Flow Batteries. Chem. Commun. 2021, 57 (24). https://doi.org/10.1039/D0CC07951K.

(24) Butler, K. T.; Davies, D. W.; Cartwright, H.; Isayev, O.; Walsh, A. Machine Learning for Molecular and Materials Science. Nature 2018, $559 \quad$ (7715), 547-555. https://doi.org/10.1038/s41586-018-0337-2.

(25) Schmidt, J.; Marques, M. R. G.; Botti, S.; Marques, M. A. L. Recent Advances and Applications of Machine Learning in Solid-State Materials Science. npj Comput. Mater. 2019, 5 (1). https://doi.org/10.1038/s41524-019-0221-0.

(26) Wei, J.; Chu, X.; Sun, X.; Xu, K.; Deng, H.; Chen, J.; Wei, Z.; Lei, M. Machine Learning in Materials Science. InfoMat 2019, 1 (3), 338-358. https://doi.org/10.1002/inf2.12028.

(27) Pilania, G.; Wang, C.; Jiang, X.; Rajasekaran, S.; Ramprasad, R. Accelerating Materials Property Predictions Using Machine Learning. Sci. Rep. 2013, 3, 1-6. https://doi.org/10.1038/srep02810.

(28) Batra, R. Accurate Machine Learning in Materials Science Facilitated by Using Diverse Data Sources. Nature 2021, 589 (7843), 524-525. https://doi.org/10.1038/d41586-020-03259-4.

(29) Gómez-Bombarelli, R.; Aguilera-Iparraguirre, J.; Hirzel, T. D.; Duvenaud, D.; Maclaurin, D.; Blood-Forsythe, M. A.; Chae, H. S.; Einzinger, M.; Ha, D. G.; Wu, T.; 
Markopoulos, G.; Jeon, S.; Kang, H.; Miyazaki, H.; Numata, M.; Kim, S.; Huang, W.; Hong, S. I.; Baldo, M.; Adams, R. P.; Aspuru-Guzik, A. Design of Efficient Molecular Organic LightEmitting Diodes by a High-Throughput Virtual Screening and Experimental Approach. Nat. Mater. 2016, 15 (10), 1120-1127. https://doi.org/10.1038/nmat4717.

(30) Hautier, G.; Fischer, C. C.; Jain, A.; Mueller, T.; Ceder, G. Finding Natures Missing Ternary Oxide Compounds Using Machine Learning and Density Functional Theory. Chem. Mater. 2010, 22 (12), 3762-3767. https://doi.org/10.1021/cm100795d.

(31) Faber, F. A.; Lindmaa, A.; Von Lilienfeld, O. A.; Armiento, R. Machine Learning Energies of 2 Million Elpasolite (ABC2D6) Crystals. Phys. Rev. Lett. 2016, 117 (13), 2-7. https://doi.org/10.1103/PhysRevLett.117.135502.

(32) Carrasquilla, J.; Melko, R. G. Machine Learning Phases of Matter. Nat. Phys. 2017, 13 (5), 431-434. https://doi.org/10.1038/nphys4035.

(33) Cavasotto, C. N.; Di Filippo, J. I. Artificial Intelligence in the Early Stages of Drug $\begin{array}{lllll}\text { Discovery. } & \text { Arch. } & \text { Biochem. } & \text { Biophys. }\end{array}$ https://doi.org/https://doi.org/10.1016/j.abb.2020.108730.

(34) Peyton, B. G.; Briggs, C.; D’Cunha, R.; Margraf, J. T.; Crawford, T. D. MachineLearning Coupled Cluster Properties through a Density Tensor Representation. J. Phys. Chem. A 2020, 124 (23), 4861-4871. https://doi.org/10.1021/acs.jpca.0c02804.

(35) Seko, A.; Hayashi, H.; Nakayama, K.; Takahashi, A.; Tanaka, I. Representation of Compounds for Machine-Learning Prediction of Physical Properties. Phys. Rev. B 2017, 95 (14), 1-11. https://doi.org/10.1103/PhysRevB.95.144110. 
(36) Sahoo, S.; Adhikari, C.; Kuanar, M.; Mishra, B. A Short Review of the Generation of Molecular Descriptors and Their Applications in Quantitative Structure Property/Activity Relationships. Curr. Comput. Aided-Drug Des. 2016, 12 (3), 181-205. https://doi.org/10.2174/1573409912666160525112114.

(37) Fisher, D.; R. Lukow, S.; Berezutskiy, G.; Gil, I.; Levy, T.; Zeiri, Y. Machine Learning Improves Trace Explosive Selectivity: Application to Nitrate-Based Explosives. J. Phys. Chem. A 2020, 124 (46), 9656-9664. https://doi.org/10.1021/acs.jpca.0c05909.

(38) Nayak, S.; Bhattacharjee, S.; Choi, J.-H.; Cheol Lee, S. Machine Learning and Scaling Laws for Prediction of Accurate Adsorption Energy. J. Phys. Chem. A 2019, 124 (1), 247-254. https://doi.org/10.1021/acs.jpca.9b07569.

(39) Wei, Y.; Chin, K.; M. Barge, L.; Perl, S.; Hermis, N.; Wei, T. Machine Learning Analysis of the Thermodynamic Responses of In Situ Dielectric Spectroscopy Data in Amino Acids and Inorganic Electrolytes. J. Phys. Chem. B 2020, 124 (50), 11491-11500. https://doi.org/10.1021/acs.jpcb.0c09266.

(40) L. Nisbet, M.; M. Pendleton, I.; M. Nolis, G.; J. Griffith, K.; Schrier, J.; Cabana, J.; J. Norquist, A.; R. Poeppelmeier, K. Machine-Learning-Assisted Synthesis of Polar Racemates. $J$. Am. Chem. Soc. 2020, 142 (16), 7555-7566. https://doi.org/10.1021/jacs.0c01239.

(41) Wexler, R. B.; Mark P. Martirez, J.; M. Rappe, A. Chemical Pressure-Driven Enhancement of the Hydrogen Evolving Activity of Ni2P from Nonmetal Surface Doping Interpreted via Machine Learning. J. Am. Chem. Soc. 2018, 140 (13), 4678-4683. https://doi.org/10.1021/jacs.8b00947. 
(42) Lee, M. H. Identification of Host-Guest Systems in Green TADF-Based OLEDs with Energy Level Matching Based on a Machine-Learning Study. Phys. Chem. Chem. Phys. 2020, 22 (28), 16378-16386. https://doi.org/10.1039/d0cp02871a.

(43) Landrum, G. RDKit: Open-source cheminformatics https://www.rdkit.org/.

(44) Ramsundar, B.; Eastman, P.; Walters, P.; Pande, V.; Leswing, K.; Wu, Z. Deep Learning for the Life Sciences; O’Reilly Media, 2019.

(45) Chaube, S.; Goverapet Srinivasan, S.; Rai, B. Applied Machine Learning for Predicting the Lanthanide-Ligand Binding Affinities. Sci. Rep. 2020, 10 (1), $1-11$. https://doi.org/10.1038/s41598-020-71255-9.

(46) A. Pugar, J.; M. Childs, C.; Huang, C.; W. Haider, K.; R. Washburn, N. Elucidating the Physicochemical Basis of the Glass Transition Temperature in Linear Polyurethane Elastomers with Machine Learning. J. Phys. Chem. $B$ 2020, 124 (43), 9722-9733. https://doi.org/10.1021/acs.jpcb.0c06439.

(47) Casey, A. D.; Son, S. F.; Bilionis, I.; Barnes, B. C. Prediction of Energetic Material Properties from Electronic Structure Using 3D Convolutional Neural Networks. J. Chem. Inf. Model. 2020, 60 (10), 4457-4473. https://doi.org/10.1021/acs.jcim.0c00259.

(48) J. Minnich, A.; McLoughlin, K.; Tse, M.; Deng, J.; Weber, A.; Murad, N.; D. Madej, B.; Ramsundar, B.; Rush, T.; Calad-Thomson, S.; Brase, J.; E. Allen, J. AMPL: A Data-Driven Modeling Pipeline for Drug Discovery. J. Chem. Inf. Model. 2020, 60 (4), 1955-1968. https://doi.org/10.1021/acs.jcim.9b01053. 
(49) Okamoto, Y.; Kubo, Y. Ab Initio Calculations of the Redox Potentials of Additives for Lithium-Ion Batteries and Their Prediction through Machine Learning. ACS Omega 2018, 3 (7), 7868-7874. https://doi.org/10.1021/acsomega.8b00576.

(50) Joshi, R. P.; Eickholt, J.; Li, L.; Fornari, M.; Barone, V.; Peralta, J. E. Machine Learning the Voltage of Electrode Materials in Metal-Ion Batteries. ACS Appl. Mater. Interfaces 2019, 11 (20), 18494-18503. https://doi.org/10.1021/acsami.9b04933.

(51) Allam, O.; Cho, B. W.; Kim, K. C.; Jang, S. S. Application of DFT-Based Machine Learning for Developing Molecular Electrode Materials in Li-Ion Batteries. RSC Adv. 2018, 8 (69), 39414-39420. https://doi.org/10.1039/c8ra07112h.

(52) Zhang, Y.; Xu, X. Machine Learning Properties of Electrolyte Additives: A Focus on Redox Potentials. Ind. Eng. Chem. Res. 2021, 60 (1), 343-354. https://doi.org/10.1021/acs.iecr.0c05055.

(53) Allam, O.; Kuramshin, R.; Stoichev, Z.; Cho, B. W.; Lee, S. W.; Jang, S. S. Molecular Structure-Redox Potential Relationship for Organic Electrode Materials: Density Functional Theory-Machine Learning Approach. Mater. Today Energy 2020, 17, 100482. https://doi.org/10.1016/j.mtener.2020.100482.

(54) Pedregosa FABIANPEDREgOSA, F.; Michel, V.; Grisel OLIVIERGRISEL, O.; Blondel, M.; Prettenhofer, P.; Weiss, R.; Vanderplas, J.; Cournapeau, D.; Pedregosa, F.; Varoquaux, G.; Gramfort, A.; Thirion, B.; Grisel, O.; Dubourg, V.; Passos, A.; Brucher, M.; Perrot andÉdouardand, M.; Duchesnay, andÉdouard; Duchesnay EDOUARDDUCHESNAY, Fré. Scikit-Learn: Machine Learning in Python Gaël Varoquaux Bertrand Thirion Vincent 
Dubourg Alexandre Passos PEDREGOSA, VAROQUAUX, GRAMFORT ET AL. Matthieu Perrot; 2011; Vol. 12.

(55) Wildman, S. A.; Crippen, G. M. Prediction of Physicochemical Parameters by Atomic Contributions. J. Chem. Inf. Comput. Sci. 1999, 39 (5), 868-873. https://doi.org/10.1021/ci9903071.

(56) Getting Started with the RDKit in Python - The RDKit 2021.03.1 documentation https://www.rdkit.org/docs/GettingStartedInPython.html\#list-of-available-descriptors (accessed Mar 31, 2021).

(57) Hall, L. H.; Mohney, B.; Kier, L. B. The Electrotopological State: Structure Information at the Atomic Level for Molecular Graphs; 1991; Vol. 31.

(58) Hall, L. H.; Kier, L. B. The Molecular Connectivity Chi Indexes and Kappa Shape Indexes in Structure-Property Modeling; John Wiley \& Sons, Ltd, 2007; pp 367-422. https://doi.org/10.1002/9780470125793.ch9.

(59) Todeschini, R.; Consonni, V. Descriptors from Molecular Geometry. In Handbook of Chemoinformatics; Wiley-VCH Verlag GmbH: Weinheim, Germany, 2008; Vol. 3, pp 10041033. https://doi.org/10.1002/9783527618279.ch37.

(60) Rogers, D.; Hahn, M. Extended-Connectivity Fingerprints. J. Chem. Inf. Model. 2010, 50 (5), 742-754. https://doi.org/10.1021/ci100050t.

(61) L. Durant, J.; A. Leland, B.; R. Henry, D.; G. Nourse, J. Reoptimization of MDL Keys for Use in Drug Discovery. J. Chem. Inf. Comput. Sci. 2002, 42 (6), 1273-1280. https://doi.org/10.1021/ci010132r. 


\section{For Table of Contents Use Only}
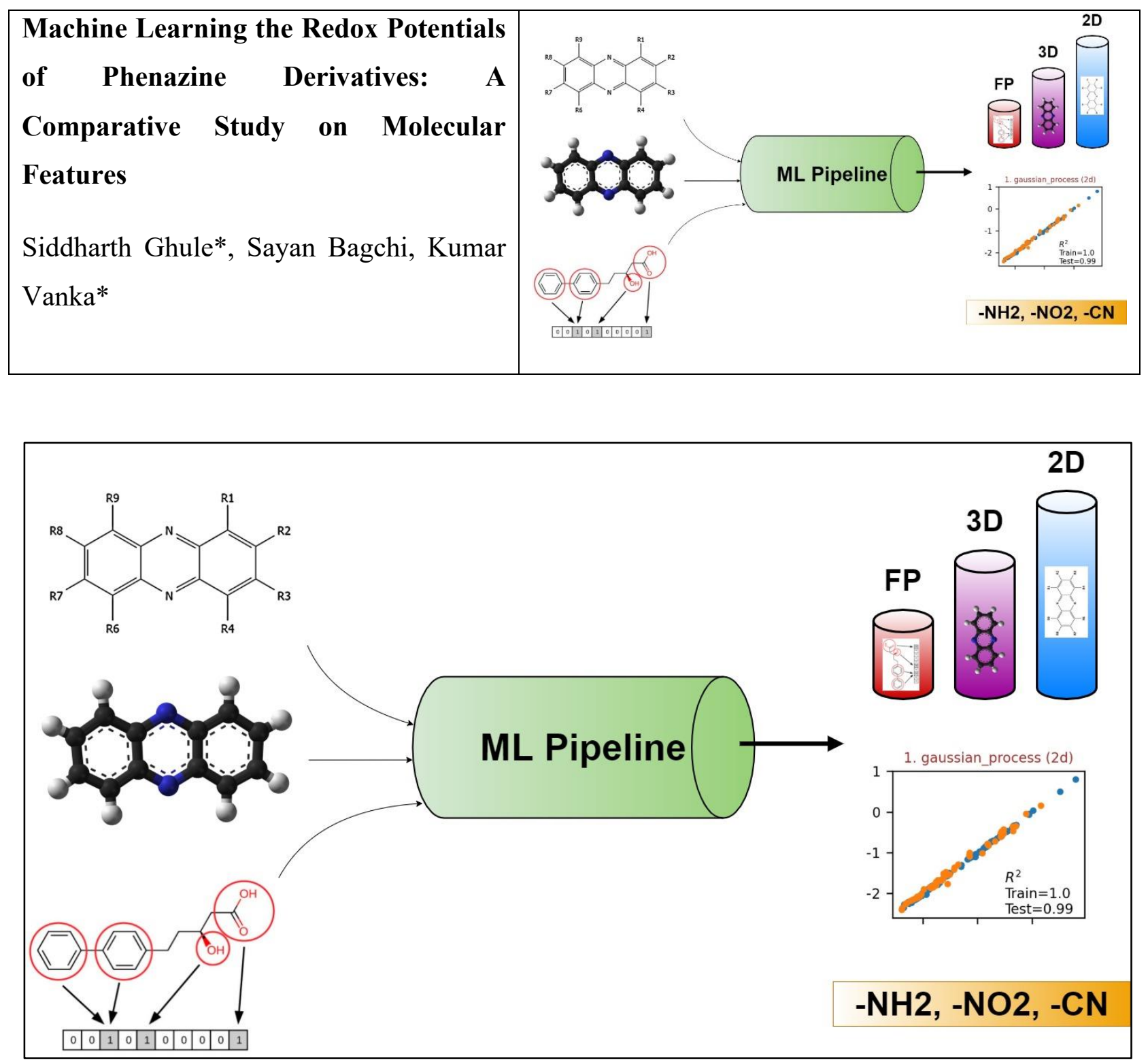